\title{
Near infrared hydrogen lines as diagnostic of accretion and winds in T Tauri stars ${ }^{\star, \star \star}$
}

\author{
D. F. M. Folha ${ }^{1}$ and J. P. Emerson ${ }^{2}$ \\ 1 Centro de Astrofísica da Universidade do Porto, Rua das Estrelas, 4150-762 Porto, Portugal \\ e-mail: dfmf@astro.up.pt \\ 2 Department of Physics, Queen Mary, University of London, Mile End Road, London E1 4NS, UK \\ e-mail: j.p.emerson@qmw.ac.uk
}

Received 5 July 2000 / Accepted 6 October 2000

\begin{abstract}
From a sample of $50 \mathrm{~T}$ Tauri stars, mostly from the Taurus-Auriga complex, $\mathrm{Pa} \beta$ line profiles were obtained for 49 of the stars and $\operatorname{Br} \gamma$ profiles for 37 of the stars. Emission at $\mathrm{Pa} \beta$ was observed for 42 stars and emission at $\operatorname{Br} \gamma$ was found for 30 stars. The most conspicuous features in the line profiles is the almost complete absence of blueshifted absorption components and the relatively high frequency of inverse $\mathrm{P}$ Cygni profiles (IPC). At $\mathrm{Pa} \beta, 34 \%$ of the profiles are IPC while at $\mathrm{Br} \gamma 20 \%$ are IPC. The redshifted absorption features indicate infall at velocities of about $200 \mathrm{~km} \mathrm{~s}^{-1}$, compatible with free fall from a few radii out. In general, line profiles are broad centrally peaked with slightly blueshifted line peaks. Existing wind and accretion models fail, in quantitative terms, to explain the shape of the observed profiles. Magnetospheric accretion models, being the currently preferred ones, produce lines too narrow (by $\sim 100 \mathrm{~km} \mathrm{~s}^{-1} F W H M$ ), wings extending to velocities too low (by at least $\sim 100 \mathrm{~km} \mathrm{~s}^{-1}$ ) and with maximum normalized intensities too high by factors of a few. A qualitative agreement between some of the accretion model predicted profiles and some observations hint that emission in these lines might, at least partially, arise from infalling material. Definite claims regarding the origin of the emission in these lines cannot be made until models match observations much better than they currently do.
\end{abstract}

Key words. line: profiles - circumstellar matter - stars: formation - stars: pre-main sequence - infrared: general - infrared: stars

\section{Introduction}

Hydrogen lines in emission are the hallmark of Classical $\mathrm{T}$ Tauri stars (CTTS). These lines tend to reflect the dynamics of the region where they are formed. Clear examples are normal or inverse $\mathrm{P}$ Cygni profiles found in the Balmer lines of many CTTS. Traditionally, the strong hydrogen emission was interpreted in terms of mass loss (Lago 1979; De Campli 1981; Hartmann et al. 1982; Natta et al. 1988; Hartmann \& Kenyon 1990). The last decade saw a shift in interpretation towards mass accretion (Calvet \& Hartmann 1992; Hartmann et al. 1994; Edwards et al. 1994; Muzerolle et al. 1998a; Muzerolle et al. 1998b), following the magnetospheric accretion

\footnotetext{
Send offprint requests to: D. F. M. Folha

* Tables 4 and 5 are only available in electronic form at the CDS via anonymous ftp to cdsarc.u-strastg.fr (130.79.128.5) or via

http://cdsweb.u-strastg.fr/cgi-bin/qcat?J/A+A/365/90

$\star \star$ Tables 6 and 7 are also available in electronic form at the CDS
}

model proposed by Camenzind (1990), Königl (1991) and Shu et al. (1994).

Considerable effort has been made in trying to model line profiles, especially those of $\mathrm{H} \alpha$, both in wind scenarios (Hartmann et al. 1990; Calvet et al. 1992; Grinin \& Mitskevich 1991; Mitskevich et al. 1993; Pedrosa 1996) and accretion scenarios (Bertout 1977; Bertout 1979; Bastian 1982; Calvet \& Hartmann 1992; Hartmann et al. 1994; Muzerolle et al. 1998a). This has been accompanied by a similar effort on the observational side, aiming at constraining the existing models (e.g. Edwards et al. 1994; Fernandez et al. 1995; Reipurth et al. 1996; Muzerolle et al. 1998b; Alencar \& Basri 2000).

A wind scenario for the formation of the hydrogen emission lines observed in $\mathrm{T}$ Tauri stars was much discussed over past years. The predominance of blueshifted absorptions in the $\mathrm{H} \alpha$ line profiles, the success in modeling some of these observations with wind models, together with the unequivocal presence of winds in CTTS has been the main driving force behind this interpretation. Despite the recent shift towards an interpretation based on the 
magnetospheric accretion model, the debate is far from settled, as it is clearly seen in Alencar \& Basri (2000). Also, for a given star, both winds and accretion flows may contribute to hydrogen line emission, with different lines being formed in distinct regions and/or affected differently by those regions.

The successes and failures of the models in explaining hydrogen line profiles in CTTS have been based on the study of lines from the Balmer series. The exception is the work by Najita et al. (1996) where the $\operatorname{Br} \gamma$ emission line is studied for a very small number of objects.

The strongest hydrogen lines in the near infrared part of the electromagnetic spectrum are lines from the Paschen and Brackett series. These lines arise in higher energy levels when compared to the lower Balmer lines (e.g. $\mathrm{H} \alpha$ and $\mathrm{H} \beta$ ) and they are generally optically thinner than the latter. One expects them to form at different depths into a cloud of hydrogen. Near infrared hydrogen lines should form in the denser parts of the circumstellar envelope of CTTS and, in particular, they should trace infalling material in a magnetospheric accretion scenario. The development of near infrared high spectral resolution high sensitivity spectroscopy allows the study of profiles of near infrared hydrogen lines. Such a study imposes strong constraints on models and contributes to the understanding of the origin of hydrogen emission in CTTS.

The structure of the paper is as follows: observations and data reduction are described in Sect. 2, the line spectra are presented in Sect. 3, classification of the line profiles is done in Sect. 4, the parameters that characterize the line profiles and their statistics are discussed in Sects. 5 and 6 . The results are compared with other observations in Sect. 7, and with models in Sect. 8. The results are discussed in Sect. 9 and conclusions are presented in Sect. 10.

\section{Observations and data reduction}

The observations were carried out at the United Kingdom Infrared Telescope (UKIRT) during two observing runs. The first took place between 2-5 October 1994 (UT), the echelle grating, long $(300 \mathrm{~mm})$ focal length camera and $58 \times 62$ pixel SBRC InSb detector of the Cooled Grating Spectrometer 4 (CGS4) were used. The second run was held between 15-17 December 1995 (UT) and CGS4 was used with the echelle grating short $(150 \mathrm{~mm})$ focal length camera and $256 \times 256$ pixel SBRC InSb detector. Spectra were obtained centered at $\mathrm{Pa} \beta(1.28215 \mu \mathrm{m})$ and at $\mathrm{Br} \gamma$ $(2.16611 \mu \mathrm{m})$ for a sample of $\mathrm{T}$ Tauri stars mainly from the Taurus-Auriga complex. The observed stars are listed in Table 1, with a " $\sqrt{ }$ " indicating whether $\mathrm{Pa} \beta$ and/or $\operatorname{Br} \gamma$ spectra was taken.

The spectral coverage is approximately $1200 \mathrm{~km} \mathrm{~s}^{-1}$ at $\mathrm{Pa} \beta$ and approximately $1400 \mathrm{~km} \mathrm{~s}^{-1}$ for spectra from the UT94 run and approximately $3600 \mathrm{~km} \mathrm{~s}^{-1}$ at $\mathrm{Pa} \beta$ and approximately $3800 \mathrm{~km} \mathrm{~s}^{-1}$ at $\operatorname{Br} \gamma$ for spectra from the UT95 run. All observed spectra were sampled twice per resolution element $(R=16000$ for UT94 and $R=20500$ for UT95). The slit size used was for the UT94 run: $1^{\prime \prime} .25 \times$ $90^{\prime \prime}$ with pixel size $1^{\prime \prime} .25$ in the dispersion direction and $2^{\prime \prime} .2$ in the spatial direction for both $\mathrm{Pa} \beta$ and $\mathrm{Br} \gamma$ setups; and for the UT95 run: $1^{\prime \prime} .2 \times 90^{\prime \prime}$ and the pixel size $1^{\prime \prime} .2$ in the dispersion direction and $1^{\prime \prime} .8$ and $1^{\prime \prime} .7$ in the spatial direction, respectively for the $\mathrm{Pa} \beta$ and $\mathrm{Br} \gamma$ wavelength regions. In both runs flat field frames were obtained for flat fielding and a number of standard stars were observed for correction of the atmospheric transmission and of the instrumental response. During the UT95 run a number of late type main sequence stars were also observed. For more details on the observational procedures and observing logs refer to Folha (1998).

The data reduction followed the general procedure described by Puxley et al. (1992) for the reduction of spectra obtained with CGS4. It was carried out using CGS4DR, Figaro and IDL routines written specifically for this work. Spectral images were masked to avoid bad pixels and vignetted areas of the detector, de-biased and flat-fielded. Sky subtraction was achieved by subtracting sky frames from object frames. The spectra were optimally extracted using Figaro's implementation of the optimal extraction algorithm developed by Horne (1986) and, when needed, de-rippled using the deripple_spectrum task in CGS4DR. CVF fringing affected spectra of the $\operatorname{Br} \gamma$ window obtained on the UT95 run and the fringing pattern was removed by filtering the spectra using standard Figaro tasks. Wavelength calibration was achieved by using $\mathrm{OH}$ airglow emission lines, telluric absorption lines and, in some cases, photospheric absorption lines. The uncertainty in the wavelength calibration is: $8-9 \mathrm{~km} \mathrm{~s}^{-1}$ at $\mathrm{Pa} \beta$ and 4-9 $\mathrm{km} \mathrm{s}^{-1}$ at Br $\gamma$ spectra from UT94 and $6-8 \mathrm{~km} \mathrm{~s}^{-1}$ at $\mathrm{Pa} \beta$ and $14-17 \mathrm{~km} \mathrm{~s}^{-1}$ at $\mathrm{Br} \gamma$ from UT95. Correction of the atmospheric transmission and instrumental response was achieved by dividing the spectra of the target stars by the spectrum of one of the observed standard stars and multiplying the result by a black body spectrum of the appropriate effective temperature. Finally, spectra had their continuum normalized to unity by dividing the spectra by a cubic spline fit to the continuum. For more details on the data reduction procedure refer to Folha (1998).

\section{The line spectra}

A number of photospheric lines are seen in absorption in the observed $\mathrm{Pa} \beta$ and $\mathrm{Br} \gamma$ spectra of several T Tauri stars (see Folha \& Emerson 1999, hereafter FE99). Some of the narrow photospheric absorption lines fall on top of the $\mathrm{Pa} \beta$ line, changing its intrinsic profile. Correct analysis of the line profiles require the photospheric component of the observed spectra to be removed. This was achieved, after computing the veiling in the observed wavelength ranges, by following the procedure described in Edwards et al. (1994) to obtain "residual" profiles (see FE99 for results on near infrared veiling).

Figures 1 and 2 show the $\mathrm{Pa} \beta$ and $\operatorname{Br} \gamma$ spectra of the observed $\mathrm{T}$ Tauri stars, arranged by the type of the line profile, from type I to type IV (as defined in Sect. 4 
Table 1. Sample of observed stars - Positions, classification (CTTS or WTTS) and spectral types from the Herbig-Bell Catalogue (1988) (HBC) and radial velocities from HBC or from the references indicated in the notes to this table. Tick marks mean that a spectrum was obtained

\begin{tabular}{|c|c|c|c|c|c|c|c|}
\hline Star & $\mathrm{RA}(1950)$ & DEC(1950) & Type & Spec.Ty. & $\overline{V_{\text {rad }}}$ & $\mathrm{Pa} \beta$ & $\mathrm{Br} \gamma$ \\
\hline V773 Tau & 4117.29 & +280441.2 & WTTS & K3 V & $+16 \mathrm{~b}$ & $\sqrt{ }$ & $\sqrt{ }$ \\
\hline FM Tau & 4117.82 & +280518.8 & CTTS & M0 & $+16:(\mathrm{i})$ & $\sqrt{ }$ & $\sqrt{ }$ \\
\hline FN Tau & 4118.61 & +282026.9 & CTTS & M5 & $+16 ;(\mathrm{ii})$ & $\sqrt{ }$ & \\
\hline CW Tau & 41111.34 & +280327.2 & CTTS & $\mathrm{K} 3 \mathrm{~V}$ & $+14.5 \mathrm{a}$ & $\sqrt{ }$ & $\sqrt{ }$ \\
\hline FP Tau & 41143.50 & +263857.5 & CTTS & $\mathrm{M} 4 \mathrm{~V}$ & $+22 \mathrm{~b}$ & $\sqrt{ }$ & $\sqrt{ }$ \\
\hline CY Tau & 41427.67 & +281328.6 & CTTS & M1 V & $+19.1 \mathrm{a}$ & $\sqrt{ }$ & \\
\hline DD Tau & 41525.10 & +280914.6 & CTTS & M1 & $+28 \mathrm{c}$ & $\sqrt{ }$ & \\
\hline Hubble 4 & 41540.89 & +281254.0 & WTTS & $\mathrm{K} 7$ & $+15.0 \mathrm{a}$ & $\sqrt{ }$ & $\sqrt{ }$ \\
\hline BP Tau & 4168.61 & +285915.3 & CTTS & K7 V & $+15.8 \mathrm{a}$ & $\sqrt{ }$ & $\sqrt{ }$ \\
\hline LkCa 7 & 41635.78 & +274228.1 & WTTS & $\mathrm{K} 7, \mathrm{M} 0 \mathrm{~V}$ & $+16.4 \mathrm{a}$ & $\sqrt{ }$ & $\sqrt{ }$ \\
\hline DE Tau & 41849.84 & +274805.2 & CTTS & M2: V & $+14.9 \mathrm{a}$ & $\sqrt{ }$ & \\
\hline RY Tau & 41850.85 & +281935.0 & CTTS & K1IV,V & +16.4 & $\sqrt{ }$ & $\sqrt{ }$ \\
\hline FS Tau & 41857.63 & +265030.5 & CTTS & M1 & +16: (iii) & $\sqrt{ }$ & \\
\hline $\mathrm{T}$ Tau & 4194.21 & +192505.4 & CTTS & KOIV,V & $+19.1 \mathrm{a}$ & $\sqrt{ }$ & $\sqrt{ }$ \\
\hline DF Tau & 42359.63 & +253541.7 & CTTS & $\mathrm{M} 0,1 \mathrm{~V}$ & $+12 \mathrm{~b}$ & $\sqrt{ }$ & $\sqrt{ }$ \\
\hline DG Tau & 4241.01 & +255935.5 & CTTS & M ? & $+16:$ (iv) & $\sqrt{ }$ & $\sqrt{ }$ \\
\hline DI Tau & 42638.00 & +262620.1 & WTTS & M0 V & $+16.0 \mathrm{a}$ & $\sqrt{ }$ & \\
\hline IQ Tau & 42647.67 & +260016.3 & CTTS & M0.5 & $+15.3 \mathrm{a}$ & $\sqrt{ }$ & \\
\hline FX Tau & 42727.91 & +242018.2 & CTTS & M1 & $+16.8 \mathrm{a}$ & $\sqrt{ }$ & \\
\hline DK Tau & 42740.48 & +255459.0 & CTTS & $\mathrm{K} 7 \mathrm{~V}$ & $+15.3 \mathrm{a}$ & $\sqrt{ }$ & $\sqrt{ }$ \\
\hline ZZ Tau & 42749.32 & +243556.9 & CTTS & M3 & $+15 ;(\mathrm{v})$ & $\sqrt{ }$ & \\
\hline HL Tau & 42844.42 & +180736.2 & CTTS & $\mathrm{K} 7, \mathrm{M} 2 ?$ & +17: (vi) & $\sqrt{ }$ & $\sqrt{ }$ \\
\hline XZ Tau & 42846.00 & +180735.2 & CTTS & M3 & $+17 ;$ (vii) & $\sqrt{ }$ & $\sqrt{ }$ \\
\hline HK Tau & 42848.85 & +241756.2 & CTTS & M0.5 & $+16.3 \mathrm{a}$ & $\sqrt{ }$ & \\
\hline Haro 6-13 & 42913.60 & +242242.9 & CTTS & Cont. & $+15 ;(\mathrm{v})$ & $\sqrt{ }$ & $\sqrt{ }$ \\
\hline GG Tau & 42937.06 & +172522.3 & CTTS & $\mathrm{K} 7 \mathrm{~V}$ & $+17.6 \mathrm{a}$ & $\sqrt{ }$ & $\sqrt{ }$ \\
\hline GH Tau & 4304.79 & +240318.3 & CTTS & $\mathrm{M} 2,3 \mathrm{~V}$ & $+18.4 \mathrm{a}$ & $\sqrt{ }$ & \\
\hline V807 Tau & 4305.2 & +240339 & CTTS & $\mathrm{K} 7 \mathrm{~V}$ & +1 (viii) & $\sqrt{ }$ & $\sqrt{ }$ \\
\hline GI Tau & 43032.33 & +24153.1 & CTTS & $\mathrm{K} 6 \mathrm{~V}$ & $+18.1 \mathrm{a}$ & $\sqrt{ }$ & $\sqrt{ }$ \\
\hline GK Tau & 43032.76 & +241452.4 & CTTS & $\mathrm{K} 7 \mathrm{~V}$ & $+18.6 \mathrm{a}$ & $\sqrt{ }$ & $\sqrt{ }$ \\
\hline DL Tau & 43036.02 & +251424.0 & CTTS & $\mathrm{K} 7 \mathrm{~V}$ & +16.0 (ix) & $\sqrt{ }$ & $\sqrt{ }$ \\
\hline AA Tau & 43153.45 & +242244.1 & CTTS & $\mathrm{K} 7 \mathrm{~V}$ & $+16.1 \mathrm{a}$ & $\sqrt{ }$ & $\sqrt{ }$ \\
\hline DN Tau & 43225.68 & +240852.3 & CTTS & M0 V & $+16.1 \mathrm{a}$ & $\sqrt{ }$ & $\sqrt{ }$ \\
\hline HP Tau & 43252.85 & +224817.7 & CTTS & K3 & $+17.7 \mathrm{a}$ & $\sqrt{ }$ & $\sqrt{ }$ \\
\hline DO Tau & 43524.18 & +26455.2 & CTTS & M0 V & $+20: c$ & $\sqrt{ }$ & $\sqrt{ }$ \\
\hline VY Tau & 43617.41 & +224202.3 & var. & M0 V & $+17.8 \mathrm{a}$ & $\sqrt{ }$ & \\
\hline DQ Tau & 44359.99 & +165440.1 & CTTS & $\mathrm{M} 0,1 \mathrm{~V}$ & $-4: c$ & $\sqrt{ }$ & \\
\hline Haro 6-37/c & 4445.90 & +165719.2 & CTTS & $\mathrm{K} 7, \mathrm{M} 0$ & $+19.5 \mathrm{~b}$ & $\sqrt{ }$ & $\sqrt{ }$ \\
\hline DR Tau & 44413.20 & +165323.8 & CTTS & Cont. & $+16.7(\mathrm{x})$ & $\sqrt{ }$ & $\sqrt{ }$ \\
\hline DS Tau & 44439.07 & +291956.2 & CTTS & K5 V & $+16.3 \mathrm{a}$ & $\sqrt{ }$ & $\sqrt{ }$ \\
\hline UY Aur & 44835.71 & +304213.6 & CTTS & $\mathrm{K} 7 \mathrm{~V}$ & $+18 \mathrm{~b}$ & $\sqrt{ }$ & $\sqrt{ }$ \\
\hline GM Aur & 45159.76 & +301714.7 & CTTS & K3 V & $+15.0 \mathrm{a}$ & $\sqrt{ }$ & $\sqrt{ }$ \\
\hline SU Aur & 45247.84 & +302919.4 & CTTS & G2 III & +16.0 & $\sqrt{ }$ & $\sqrt{ }$ \\
\hline RW Aur A & 5437.69 & +302013.9 & CTTS & $\mathrm{K} 1$ & $+14: \mathrm{a}$ & $\sqrt{ }$ & $\sqrt{ }$ \\
\hline GW Ori & 52620.78 & +114952.8 & CTTS & G5 & $+33.6 \mathrm{a}$ & $\sqrt{ }$ & $\sqrt{ }$ \\
\hline YY Ori & 53220.77 & -55952.7 & CTTS & $\mathrm{K} 5 \mathrm{~V}$ & $+12: \mathrm{c}$ & $\sqrt{ }$ & \\
\hline TW Hya & 105930.08 & -342607.4 & CTTS & $\mathrm{K} 7 \mathrm{~V}$ & $+6: c$ & & $\sqrt{ }$ \\
\hline V1331 Cyg & 205932.21 & +500955.5 & CTTS & Cont. & $-15:(x i)$ & $\sqrt{ }$ & $\sqrt{ }$ \\
\hline DI Cep & 22548.18 & +582359.5 & CTTS & G8 V & $-10 \mathrm{~b}$ & $\sqrt{ }$ & $\sqrt{ }$ \\
\hline BM And & 233512.41 & +480735.9 & CTTS & $\mathrm{K} 5 \mathrm{~V}$ & -15.2 (xii) & $\sqrt{ }$ & $\sqrt{ }$ \\
\hline
\end{tabular}

Notes: (i) $V_{\mathrm{MC}}(\mathrm{LSR}) \sim+7 \mathrm{~km} \mathrm{~s}^{-1}$ from Levreault (1988); (ii) ad hoc velocity equal to that used for FM Tau; (iii) $V_{\mathrm{MC}}(\mathrm{LSR}) \sim$ $+7 \mathrm{~km} \mathrm{~s}^{-1}$ from Levrault (1988); (iv) $V_{\mathrm{MC}}(\mathrm{LSR})=+6.5 \mathrm{~km} \mathrm{~s}^{-1}$ from $\mathrm{C}^{18} \mathrm{O}$ data in Kitamura et al. (1996); (v) ad hoc velocity, typical of stars in Taurus; (vi) Edwards et al. (1987); (vii) ad hoc velocity equal to that used for HL Tau; (viii) Neuhäuser et al. (1995); (ix) SIMBAD database and reference therein; (x) Guenther \& Hessman (1993a); (xi) Najita et al. (1996) ; (xii) Guenther \& Hessman (1993b). A colon (:) means that the radial velocity is taken from molecular line data and a semi-colon (;) means that the radial velocity is a guess. $V_{\mathrm{MC}}(\mathrm{LSR})$ is the radial velocity determined by molecular observations, such as CO. 

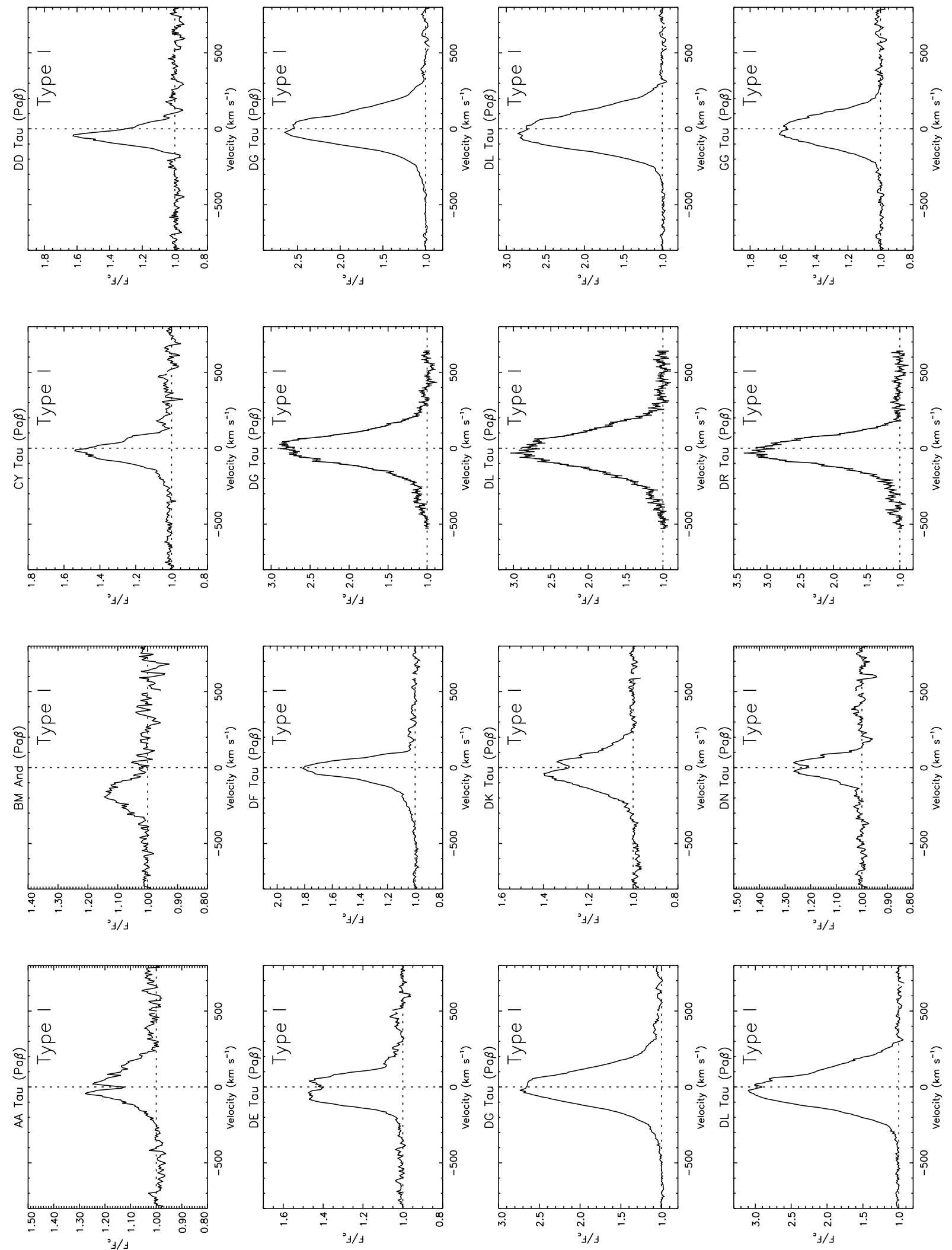

Fig. 1. $\mathrm{Pa} \beta$ line profiles. Spectra are presented by the type of the line profile and for each type by alphabetical order. Spectra with error bars in each spectral point are from the UT9410 observing run. Stars for which more than one line profile is presented have the lines arranged by observing date 

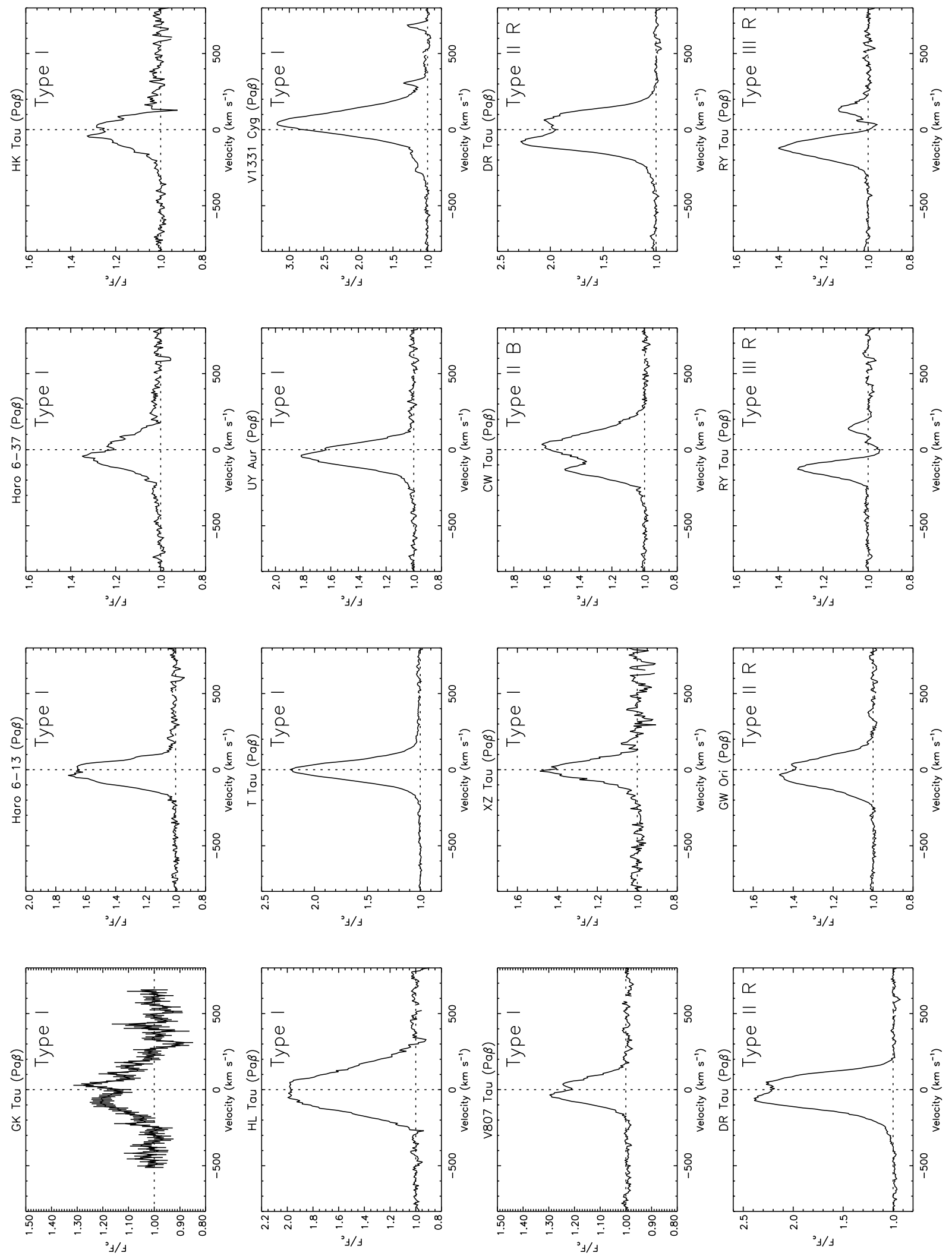

Fig. 1. continued 

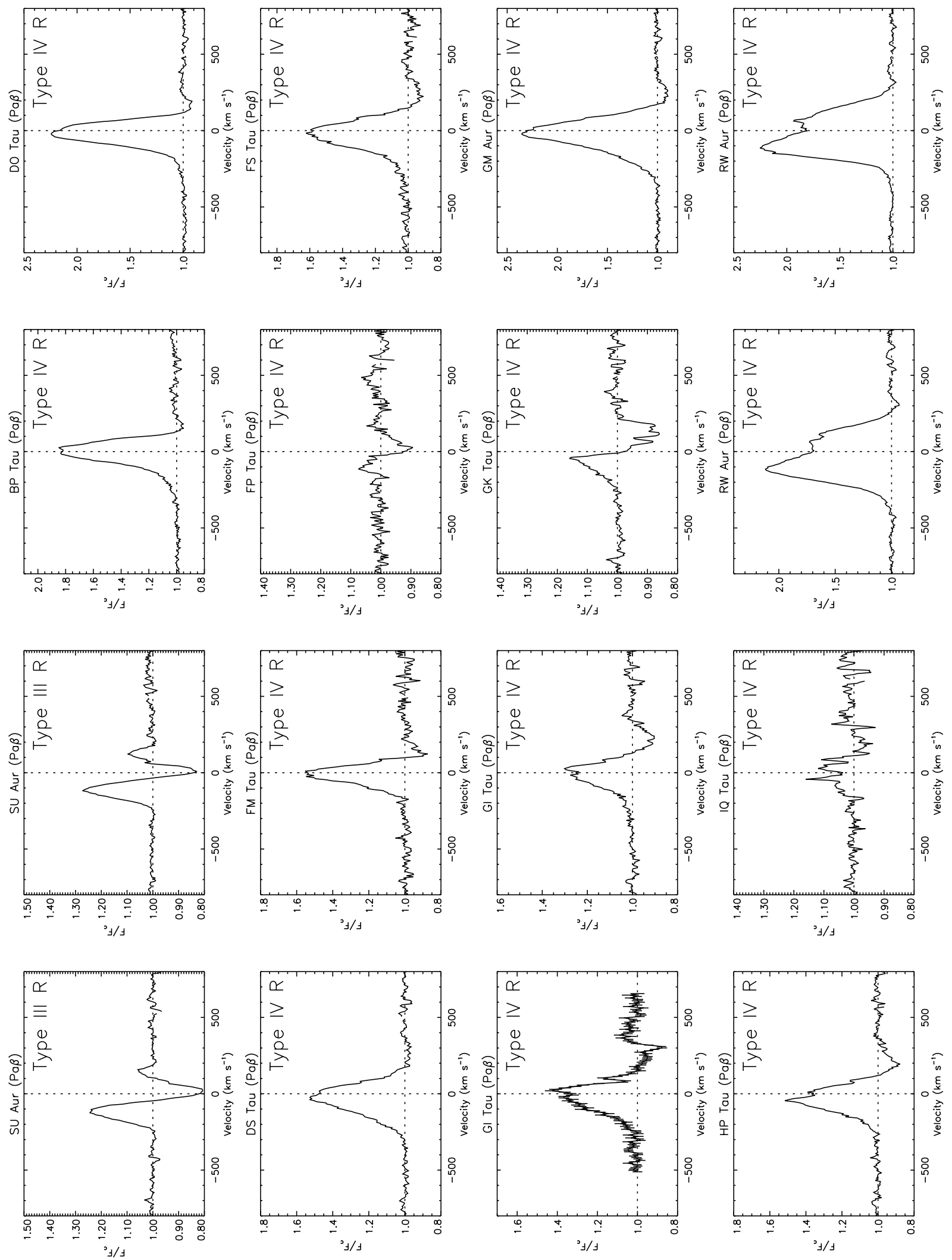

Fig. 1. continued 

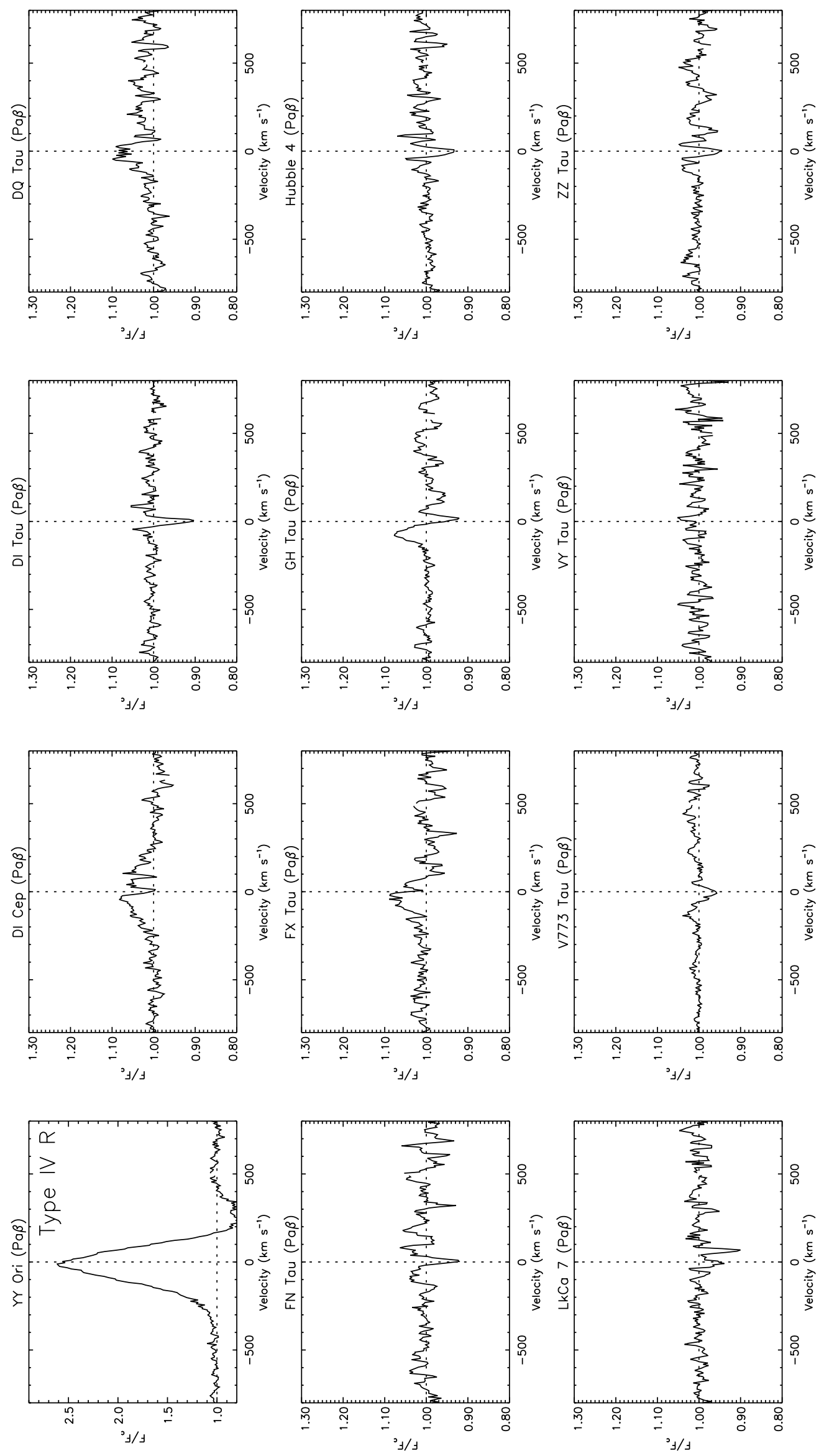

Fig. 1. continued 
Table 2. Classification of the $\mathrm{Pa} \beta$ emission line profiles according to the classification scheme developed by Reipurth et al. (1996) for the observed T Tauri stars

\begin{tabular}{|c|c|c|c|}
\hline Type & $\%$ & No. Stars & Star name \\
\hline $\mathrm{I}$ & $53 \%$ & 20 & $\begin{array}{c}\text { AA Tau BM And CY Tau } \\
\text { DD Tau DE Tau DF Tau } \\
\text { DG Tau DK Tau DL Tau } \\
\text { DN Tau GG Tau Haro 6-13 } \\
\text { Haro 6-37 HL Tau HK Tau } \\
\text { T Tau UY Aur V1331 Cyg } \\
\text { V807 Tau XZ Tau }\end{array}$ \\
\hline II B & $3 \%$ & 1 & CW Tau \\
\hline II R & $5 \%$ & 2 & DR Tau GW Ori \\
\hline III B & $\overline{0 \%}$ & 0 & - \\
\hline III $\mathrm{R}$ & $5 \%$ & 2 & RY Tau SU Aur \\
\hline IV B & $0 \%$ & 0 & - \\
\hline IV R & $34 \%$ & 13 & $\begin{array}{c}\text { BP Tau DO Tau DS Tau } \\
\text { FM Tau FP Tau FS Tau } \\
\text { GI Tau GK Tau GM Aur } \\
\text { HP Tau IQ Tau RW Aur } \\
\text { YY Ori }\end{array}$ \\
\hline
\end{tabular}

below) and for each type by alphabetical order of the name of the star. For the stars for which the veiling could be determined (FE99) the line profiles presented here are "residual" profiles, as defined by Edwards et al. (1994). The small number of profiles obtained in UT94 are not "residual" profiles, as veiling measurements were not possible with that data set.

Inspecting the line profiles one concludes that of the 49 stars observed at $\mathrm{Pa} \beta, 42$ show the line in emission and of the 36 stars observed at $\operatorname{Br} \gamma, 30$ show the line in emission. The stars without $\mathrm{Pa} \beta$ in emission are: DI Tau, FN Tau, Hubble 4, LkCa 7, V773 Tau, VY Tau and ZZ Tau, of which DI Tau, Hubble 4, LkCa 7 and V773 Tau are classified as Weak Line T Tauri stars (hereafter WTTS) in the Herbig \& Bell Catalogue (Herbig \& Bell 1988). The stars without $\mathrm{Br} \gamma$ in emission are: DN Tau, FP Tau, GI Tau, Hubble 4, LkCa 7 and V807 Tau of which Hubble 4 and LkCa 7 are WTTS.

From the stars observed both at $\mathrm{Pa} \beta$ and $\mathrm{Br} \gamma$ some display emission in one line but not in the other. Those with emission at $\mathrm{Pa} \beta$ but not at $\mathrm{Br} \gamma$ are DN Tau, FP Tau, GI Tau and V807 Tau. V773 Tau displays emission at Br $\gamma$ but not at $\mathrm{Pa} \beta$. While the former is not surprising, the latter would be puzzling if it were not for the highly variable characteristics of V773 Tau (Feigelson et al. 1994).

The lines that appear in emission display a variety of profiles. Although no two profiles are the same, many show similar properties and it is useful to separate them according to the general shape of the line profile.

\section{Line profile classification}

\subsection{Classification scheme}

What procedure should be used to classify the line profiles? Reipurth et al. (1996) review, briefly, existing classification schemes for the $\mathrm{H} \alpha$ line and propose a new one,
Table 3. Classification of the Br $\gamma$ emission line profiles according to the classification scheme developed by Reipurth et al. (1996) for the T Tauri stars

\begin{tabular}{cccc}
\hline \hline Type & $\%$ & No. Stars & Star name \\
\hline I & $72 \%$ & 18 & BP Tau DG Tau DI Cep \\
& & & DL Tau DO Tau DR Tau \\
& & & FM Tau GG Tau GM Aur \\
& & & GW Ori Haro 6-13 HL Tau \\
& & & T Tau TW Hya UY Aur \\
& & & V1331 Cyg V773 Tau XZ Tau \\
\hline II B & $0 \%$ & 0 & RY Tau SU Aur \\
II R & $8 \%$ & 2 & - \\
\hline III B & $0 \%$ & 0 & - \\
III R & $0 \%$ & 0 & BM And CW Tau DF Tau \\
IV B & $0 \%$ & 0 & HP Tau RW Aur \\
II R & $20 \%$ & 5 & \\
& & &
\end{tabular}

which tries to reflect an underlying physical process as well as being able to deal with a greater variety of line profiles. Given that all $\mathrm{Pa} \beta$ and $\mathrm{Br} \gamma$ emission line profiles displayed in Figs. 1 and 2 fit in one of the classes of that classification scheme there is no need to introduce yet another one. Furthermore, this will allow a straightforward comparison between what is found for $\mathrm{H} \alpha$ and what is found for these near infrared lines.

The Reipurth et al. (1996) classification scheme divides the line profiles into four main types: type I profiles are generally symmetric showing no evidence for absorption features or only very slight influence from those; type II profiles show two peaks with the intensity of the second peak exceeding half the strength of the main peak; type III profiles show two peaks with the intensity of the second peak being less than half the strength of the main peak and finally type IV profiles, which show an absorption feature beyond which no emission is seen. To types II, III and IV the letters B or R are appended, depending on the location of the secondary peak/absorption feature relative to the main peak: if blueshifted a B is appended, if redshifted an $\mathrm{R}$ is added. Note that type IV $\mathrm{B}$ correspond to normal $\mathrm{P}$ Cygni profiles and type IV R correspond to inverse P Cygni profiles (henceforth IPC).

\subsection{Results}

The classification of the $\operatorname{Pa} \beta$ and $\operatorname{Br} \gamma$ (hereafter referred to as NIR lines) line profiles displayed in Figs. 1 and 2 is presented in Tables 2 and 3 respectively. Some of the NIR lines observed to be in emission are too noisy too allow a reliable classification of their profiles: DI Cep, DQ Tau, FX Tau and GH Tau in Pa $\beta$ lines; and AA Tau, DK Tau, DS Tau, GK Tau and Haro 6-37 in Br $\gamma$ lines. These were not considered for the statistics presented in Tables 2 and 3 .

The main conclusions that can be taken from Tables 2 and 3 are the following: most line profiles are generally symmetric, especially the $\operatorname{Br} \gamma$ line profiles, where $72 \%$ of the profiles are classified as type I; both $\mathrm{Pa} \beta$ and 

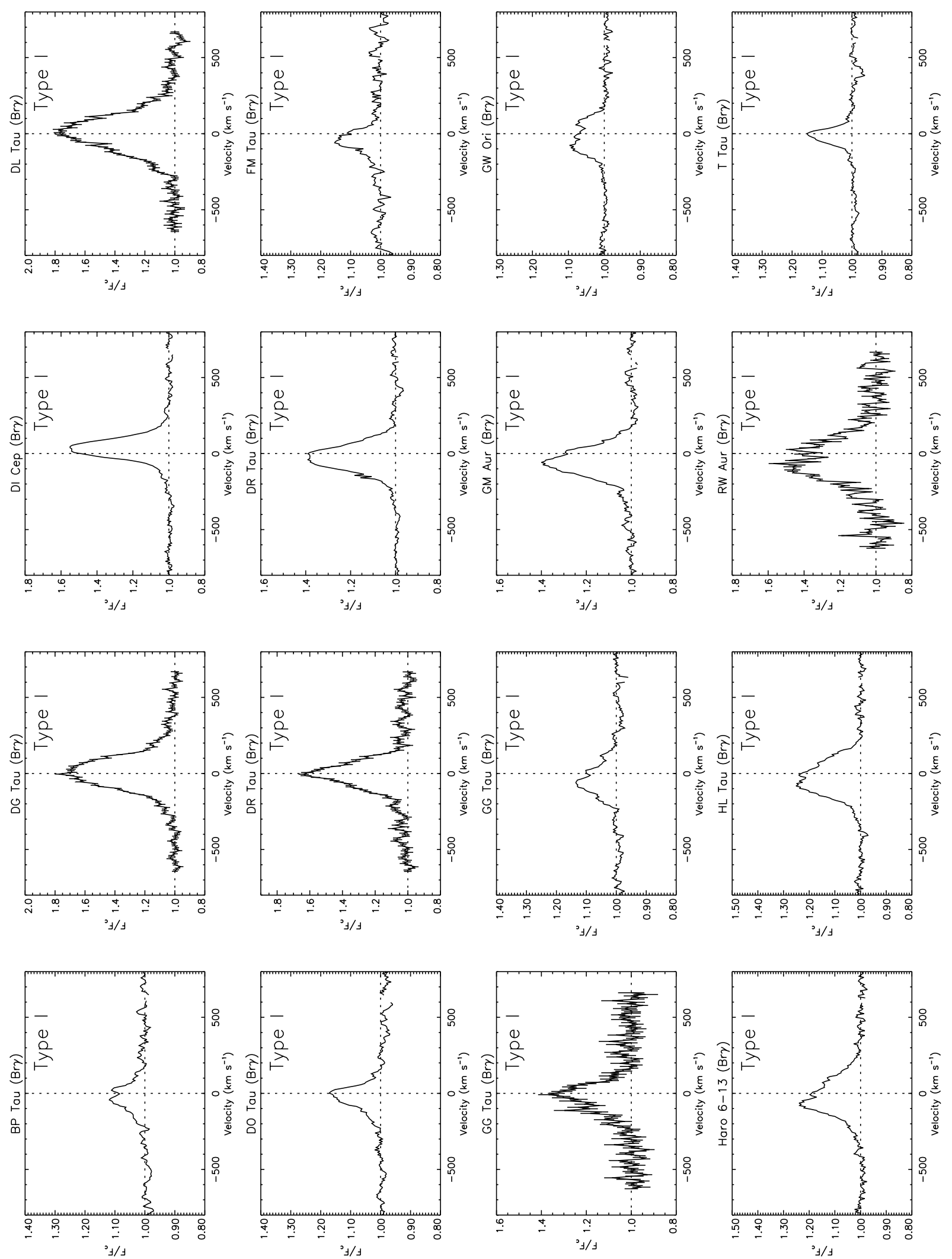

Fig. 2. Br $\gamma$ line profiles. Spectra are presented by the type of the line profile and for each type by alphabetical order. Spectra with error bars in each spectral point are from the UT9410 observing run. Stars for which more than one line profile is presented have the lines arranged by observing date 

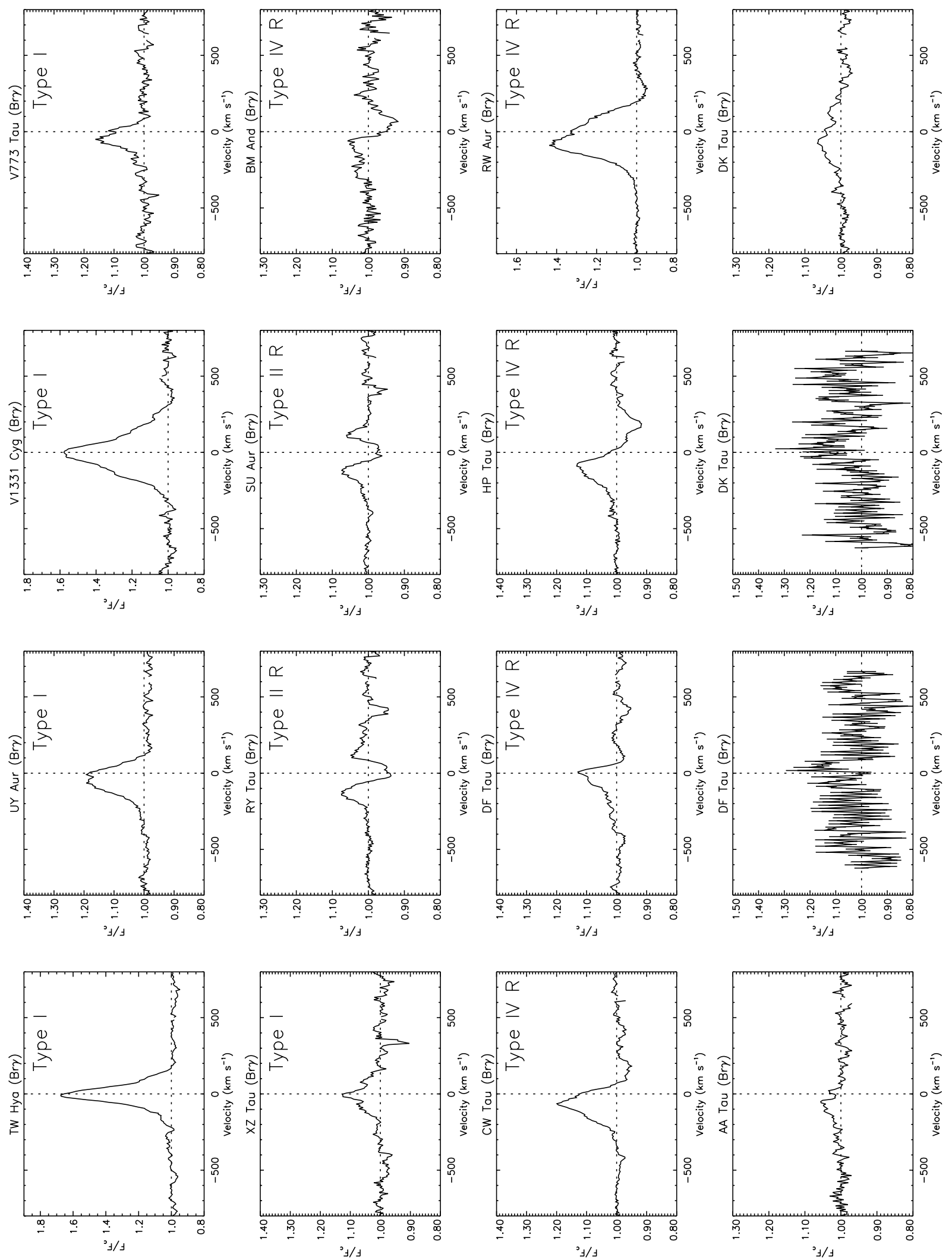

Fig. 2. continued 

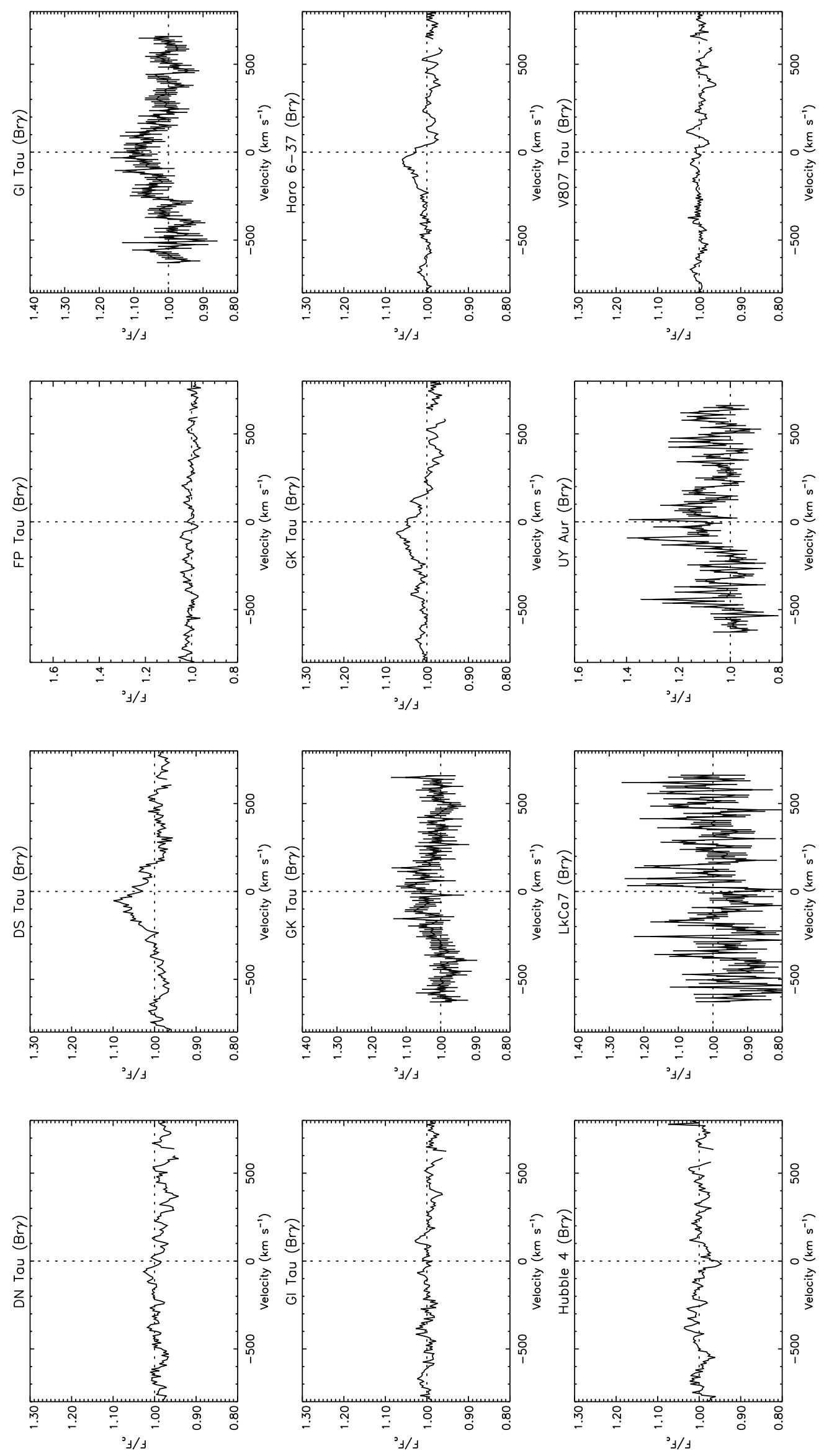

Fig. 2. continued 
Table 6. $\mathrm{Pa} \beta$ inverse $\mathrm{P}$ Cygni line profiles - Equivalent widths of the emission and of the absorption (measured below the continuum) component, central velocity of the absorption feature and line to continuum ratio $(L / C)$ at the bottom of the absorption feature

\begin{tabular}{ccccc}
\hline \hline Star & $\begin{array}{c}E W_{\text {emission }} \\
\left(\mathrm{km} \mathrm{s}^{-1}\right)\end{array}$ & $\begin{array}{c}E W_{\text {absorption }} \\
\left(\mathrm{km} \mathrm{s}^{-1}\right)\end{array}$ & $\begin{array}{c}V_{\text {abs }} \\
\left(\mathrm{km} \mathrm{s}^{-1}\right)\end{array}$ & $L / C$ \\
BP Tau & $-147.3 \pm 0.7$ & $4.8 \pm 0.1$ & 170 & 0.96 \\
DO Tau & $-231.0 \pm 0.6$ & $7.4 \pm 0.1$ & 175 & 0.93 \\
DS Tau & $-118.5 \pm 0.4$ & $2.5 \pm 0.1$ & 275 & 0.97 \\
FM Tau & $-81.9 \pm 0.6$ & $6.0 \pm 0.2$ & 150 & 0.92 \\
FS Tau & $-127.4 \pm 0.7$ & $8.3 \pm 0.4$ & 250 & 0.92 \\
FP Tau & $-3.4 \pm 0.4$ & $11.0 \pm 0.2$ & 45 & 0.93 \\
GI Tau & $-56.6 \pm 0.6$ & $11.0 \pm 0.3$ & 240 & 0.91 \\
GK Tau & $-20.7 \pm 0.3$ & $17.0 \pm 0.1$ & 115 & 0.88 \\
GM Aur & $-298.5 \pm 0.6$ & $13.2 \pm 0.2$ & 250 & 0.90 \\
HP Tau & $-92.8 \pm 0.4$ & $14.0 \pm 0.1$ & 200 & 0.91 \\
IQ Tau & $-18.6 \pm 0.5$ & $2.6 \pm 0.2$ & 200 & 0.97 \\
RW Aur & $-332.6 \pm 0.4$ & $2.8 \pm 0.1$ & 300 & 0.95 \\
YY Ori & $-357 \pm 1$ & $30.8 \pm 0.3$ & 280 & 0.81 \\
\hline \hline
\end{tabular}

$\operatorname{Br} \gamma$ line profiles lack blueshifted absorptions (only one star - CW Tau - displays any sort of absorption in the blue wing and only in $\mathrm{Pa} \beta$ ); nearly one third of the $\mathrm{Br} \gamma$ profiles have redshifted absorptions, with this number being even higher for the $\mathrm{Pa} \beta$ profiles, of which $44 \%$ display redshifted absorptions.

\subsection{Remarks}

From Figs. 1 and 2 it can be seen that some profiles classified as type I seem to show an absorption feature on or near the line centre. These absorptions could have lead us to classify those profiles either as type II B or II R. However, it should be noted that stars with no line emission also tend to show such an absorption in the "residual" profile (e.g. Hubble 4, DI Tau). These absorptions seem to be due to the fact that the main sequence dwarves used to remove the photospheric component of the spectra, do not accurately represent the T Tauri stars' photospheric spectrum for $\mathrm{Pa} \beta$ and $\mathrm{Br} \gamma$ themselves, even though the strength of the metallic lines is well matched. That is, $\mathrm{Pa} \beta$ and $\mathrm{Br} \gamma$ are deeper in the $\mathrm{T}$ Tauri photospheric spectrum than in the main sequence dwarves. When we use WTTS, such as Hubble 4, as photospheric templates, the absorption we are referring to tend to disappear, strengthening our interpretation above. Despite this, we still decided to use the main sequence dwarves as photospheric templates. The reasons for this are discussed in FE99. Quantification of this "excess" strength for the photospheric component and its interpretation is beyond the scope of this paper and will be tackled in future work.

For a few stars the $\mathrm{Pa} \beta$ and/or $\mathrm{Br} \gamma$ lines were observed more than once (refer to Sect. 9.4). The classification presented above refers to data obtained during the second observing run, i.e. December 1995, only. This is justified since, with the exception of the WTTS LkCa 7, all stars
Table 7. Br $\gamma$ inverse P Cygni line profiles - Equivalent widths of the emission and of the absorption (measured below the continuum) component, central velocity of the absorption feature and line to continuum ratio $(L / C)$ at the bottom of the absorption feature

\begin{tabular}{ccccc}
\hline \hline Star & $\begin{array}{c}E W_{\text {emission }} \\
\left(\mathrm{km} \mathrm{s}^{-1}\right)\end{array}$ & $\begin{array}{c}E W_{\text {absorption }} \\
\left(\mathrm{km} \mathrm{s}^{-1}\right)\end{array}$ & $\begin{array}{c}V_{\text {abs }} \\
\left(\mathrm{km} \mathrm{s}^{-1}\right)\end{array}$ & $L / C$ \\
BM And & $-9.3 \pm 0.4$ & $8.4 \pm 0.2$ & 60 & 0.94 \\
CW Tau & $-31.2 \pm 0.2$ & $4.3 \pm 0.1$ & 160 & 0.96 \\
DF Tau & $-18.2 \pm 0.2$ & $3.3 \pm 0.1$ & 120 & 0.98 \\
HP Tau & $-24.8 \pm 0.2$ & $11.5 \pm 0.1$ & 185 & 0.92 \\
RW Aur & $-109.0 \pm 0.3$ & $5.6 \pm 0.1$ & 275 & 0.96 \\
\hline \hline
\end{tabular}

observed during UT94 were also observed during the 1995 campaign but with much higher signal-to-noise.

The subject of variability in the line profiles will be left for Sect. 9.4, however a few remarks are relevant to the present discussion. The amount of variation present in the $\mathrm{Pa} \beta$ line profile of the stars observed twice during the 1995 campaign is such that the type of line profile did not change. However, for some stars, significant changes are seen in spectra taken 14 months apart, i.e. in October 1994 and in December 1995. These changes do not significantly alter the statistics presented, however they should be borne in mind.

\section{Line parameters}

Having a set of reliable parameters characterizing a given spectral line is of importance for the understanding of the physical conditions in the region where the line is formed. The following parameters were computed for the emission lines presented in this work: line to continuum ratio at emission line peak and velocity at which it occurs $\left(v_{\text {peak }}\right)$, full width at half maximum $(F W H M)$, half width at zero intensity $(H W Z I)$, maximum velocities observed in the line wings $\left(v_{\text {blue }}\right.$ and $\left.v_{\text {red }}\right)$, equivalent width $(E W)$ and Asymmetry Factor $(A f)$. The $H W Z I$ was computed from $v_{\text {blue }}$ and $v_{\text {red }}$ which were calculated at the $2 \%$ level of the the peak intensity. The name " $H W Z I$ " is therefore slightly misleading since, in reality, the width was not computed at zero intensity. However, " $H W Z I$ " will be used throughout this work. The $A f$ is defined as the ratio of the line equivalent width blueward of the zero velocity to the line equivalent width redward of the zero velocity.

A method devised to measure these parameters and to estimate the associated uncertainties is described in Folha (1998).

\subsection{Line parameters: Results}

The results obtained for the $\mathrm{T}$ Tauri stars' $\mathrm{Pa} \beta$ and $\mathrm{Br} \gamma$ line parameters are presented in Tables ?? to 7. Tables 6 and 7 show results of parameters specific to type IV R line profiles, such as the $E W$ of the emission and absorption components, the central velocity of the redshifted absorption feature and the line-to-continuum ratio at the bot- 

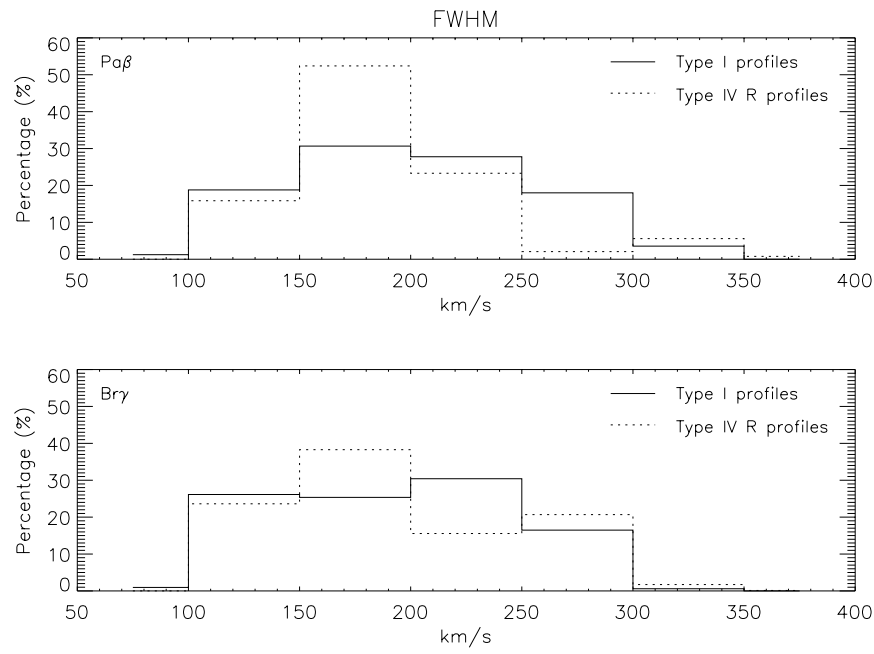

Fig. 3. Top panel: distribution of the Full Width at Half Maximum for $\mathrm{Pa} \beta$ type I (solid line) and type IV R (dotted line) profiles. Bottom panel: same as top panel for $\mathrm{Br} \gamma$

tom of the absorption. Before proceeding, a point should be made about the impact of the wavelength calibration uncertainties in some of the parameters presented in Tables ?? and ??. Those uncertainties, presented in column 3 of those tables, affect mostly the values of $v_{\text {peak }}$ and Af. The uncertainties quoted in Tables ?? and ?? for $v_{\text {peak }}$ are often similar to or smaller than the wavelength calibration uncertainty. The uncertainty quoted for $A f$ in Tables ?? and ?? results only from the noise in the spectrum. There is, however, an uncertainty in the zero velocity that results from the wavelength calibration. Such an uncertainty is propagated into $A f$ via $E W_{\text {blue }}$ and $E W_{\text {red }}$. The final relative uncertainty in $A f$ can be of the order of a few tenths. These two points should be kept in mind in the remainder of this paper.

\section{Statistics}

\subsection{General characteristics}

The lines are very broad, with mean FWHM of $204 \pm$ $12 \mathrm{~km} \mathrm{~s}^{-1}$ for $\mathrm{Pa} \beta$ and $207 \pm 26 \mathrm{~km} \mathrm{~s}^{-1}$ for $\mathrm{Br} \gamma$. Line peaks are, generally, slightly blueshifted and very rarely redshifted. The blueshift is, in most cases, very slight, only seldom exceeding $60 \mathrm{~km} \mathrm{~s}^{-1}$ for $\mathrm{Pa} \beta$ and $90 \mathrm{~km} \mathrm{~s}^{-1}$ for $\operatorname{Br} \gamma$. In fact, most lines have their peaks located within $20 \mathrm{~km} \mathrm{~s}^{-1}$ of the rest velocity.

Typical parameters for each type of line profiles and comparison between them are discussed in the following sections.

\subsection{The type I and type IV $R$ line profiles}

Type I lines are broad, with $F W H M$ ranging between 100 and $300 \mathrm{~km} \mathrm{~s}^{-1}$ (Fig. 3), and slightly blueshifted but seldom redshifted (Fig. 4). The $\mathrm{Pa} \beta$ type I lines are nearly symmetric, with $A f$ s mostly falling between 1 and 1.2 (Fig. 5). The $\operatorname{Br} \gamma$ type I line profiles are slightly more
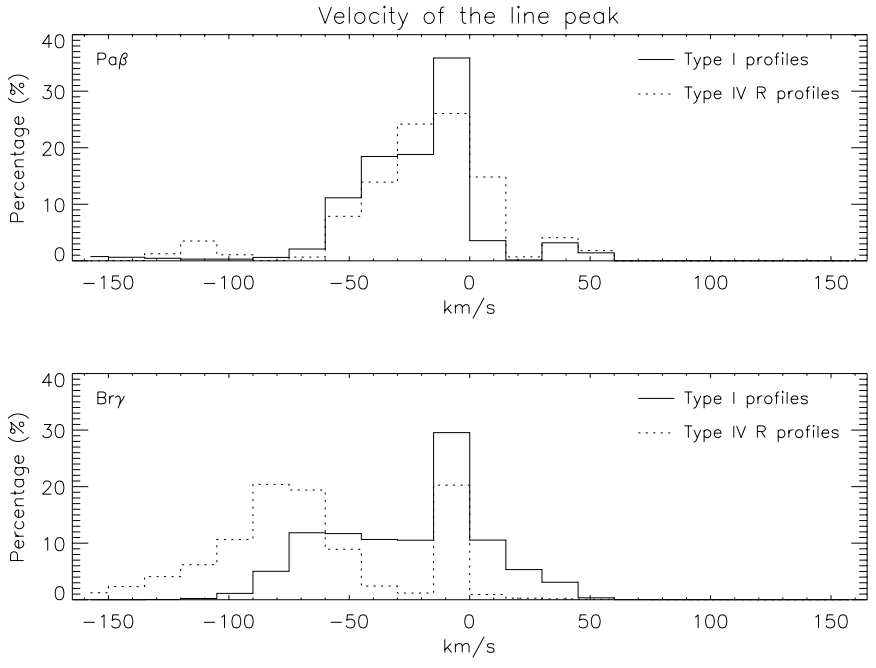

Fig. 4. Top panel: distribution of the line peak velocity for $\mathrm{Pa} \beta$ type I (solid line) and type IV R (dotted line) profiles. Bottom panel: same as top panel for $\mathrm{Br} \gamma$
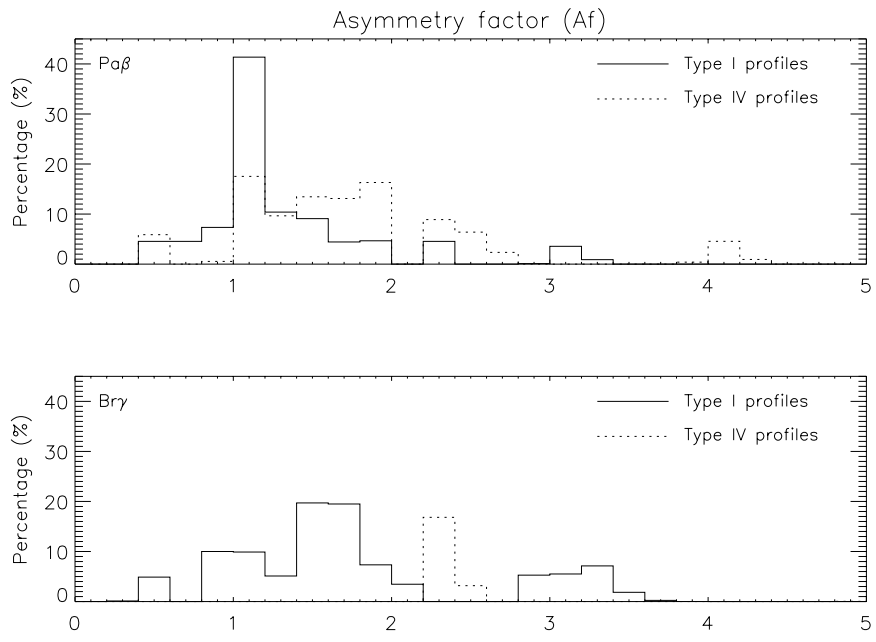

Fig. 5. Top panel: distribution of the asymmetry factor, $A f$, for $\mathrm{Pa} \beta$ type I (solid line) and type IV $\mathrm{R}$ (dotted line) profiles. Bottom panel: same as top panel for $\mathrm{Br} \gamma$

asymmetric, with $A$ fs concentrated more between 1.4 and 1.8 (Fig. 5), as well as towards a value of 3 .

The distributions of the maximum velocities observed in the blue and red wings of type I profiles, are shown in Fig. 6 as solid lines. The average maximum velocities observed are $282 \mathrm{~km} \mathrm{~s}^{-1}$ and $240 \mathrm{~km} \mathrm{~s}^{-1}$ respectively for the blue and red wings of the $\mathrm{Pa} \beta$ profiles. The equivalent values for the $\operatorname{Br} \gamma$ profiles are, respectively $305 \mathrm{~km} \mathrm{~s}^{-1}$ (blue wing) and $208 \mathrm{~km} \mathrm{~s}^{-1}$ (red wing). Hence, while $\mathrm{Pa} \beta$ type I profiles tend to be symmetric, $\mathrm{Br} \gamma$ profiles tend to show less extended red wings (by about $100 \mathrm{~km} \mathrm{~s}^{-1}$ ).

Type IV R line profiles correspond to inverse P Cygni (IPC) profiles and therefore are characterized by the presence of a redshifted absorption feature. Typical velocities for these are between $50 \mathrm{~km} \mathrm{~s}^{-1}$ and $300 \mathrm{~km} \mathrm{~s}^{-1}$, while their $E W$ s can be as low as $2.5 \mathrm{~km} \mathrm{~s}^{-1}$ for DS Tau's $\mathrm{Pa} \beta$ profile and as large as $30.8 \mathrm{~km} \mathrm{~s}^{-1}$ for YY Ori's $\mathrm{Pa} \beta$ profile. 

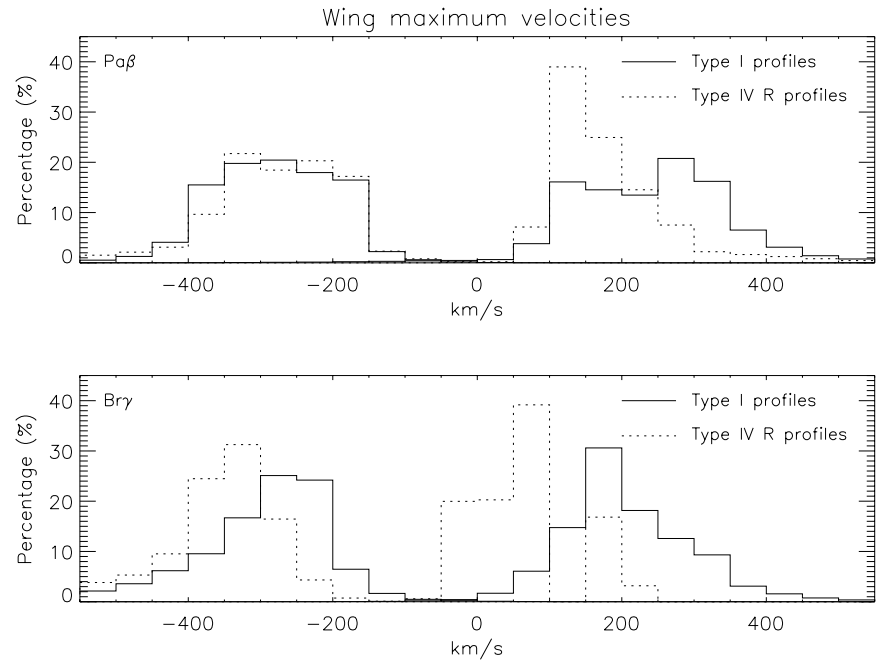

Fig. 6. Distribution of the maximum velocities seen in the line wings. Top panel: data for $\mathrm{Pa} \beta$ type I (solid lines) and type IV R (dotted lines) profiles; Bottom panel: same as top panel for $\operatorname{Br} \gamma$

For an easier comparison between the observed properties of type IV R and of type I line profiles (which constitute the majority of the profiles), the distributions of the various observed parameters for IPC profiles are plotted with those corresponding to type I profiles in Figs. 4 to 6.

The observational characteristics of IPC profiles are similar to those of type I profiles except for factors related to the redshifted absorption, most notably apparent in the Af and the maximum velocity observed for the red wing of the profile, as can be clearly seen in Figs. 5 and 6 . For IPC $\mathrm{Pa} \beta$ lines, the median $A f$ is 1.67 (cf. median $A f=1.16$ for type I $\mathrm{Pa} \beta$ profiles). For $\mathrm{Br} \gamma$ IPC lines, only one (in five) has a value for $A f$ in the interval 0 to 5 (see Fig. 5 ). Of the remaining IPC profiles, three have negative values for Af (due to the larger equivalent width of the absorption than that of the emission redward of the rest velocity) and one has an $A f$ of around 5.8. Also, the red wing in IPC profiles extends to less than $200 \mathrm{~km} \mathrm{~s}^{-1}$ for about $60 \%$ of the lines. In contrast, type I profiles have red wings extending to such low velocities for less than $35 \%$ of the lines.

The line widths of IPC and type I profiles, as measured by the FWHM, are not significantly different. However, the former tend to be narrower than the latter, with a larger percentage of stars of the former type occupying the 150 to $200 \mathrm{~km} \mathrm{~s}^{-1}$ bin in Fig. 3. Also, the distributions of the line peak velocity for IPC and type I profiles are similar, with a significant percentage of profiles peaking just blueward of the system's rest velocity (see Fig. 4). However, one should note that unlike the distribution for type I Br $\gamma$ profiles, more than half of the $\mathrm{Br} \gamma$ IPC profiles have their line peaks occurring between about $60 \mathrm{~km} \mathrm{~s}^{-1}$ and $100 \mathrm{~km} \mathrm{~s}^{-1}$ (Fig. 4). Finally, the distributions of the maximum velocity observed at the blue wing of both IPC and type I profiles are similar, with that for $\mathrm{Br} \gamma$ IPC profiles slightly shifted to higher velocities (Fig. 6).

\subsection{The type II and type III line profiles}

The number of emission lines classified as either type II or type III is small. For emission lines of these classes, histograms of the various line parameters were therefore not computed.

The emission lines with these types of profiles have characteristics similar to those displayed by type I profiles. Differences arise due to the presence of the blueshifted absorption feature in CW Tau (type II B) and due to the presence of the redshifted absorption feature in the remaining stars (types II R and III R). These absorptions change mainly the asymmetry factors, which become larger for types II $\mathrm{R}$ and III $\mathrm{R}$ and smaller for type II B relatively to $A f$ s in type I profiles.

In conclusion, all types of observed line profiles have broadly similar characteristics. Most of the differences in their properties, as indicated by the various measured parameters, seem to result from the presence of absorption features (e.g. blueshifted in CW Tau and redshifted in IPC profiles) superimposed on the common emission. The blue wing of all line profiles (the least affected by absorption features) have similar characteristics (width, maximum observed velocity) independently of the type of line profile. This seems to point to a common origin of the emission observed in the various types of profiles.

\section{Comparison with other observations \\ 7.1. NIR data}

High resolution spectroscopy of near infrared hydrogen lines in $\mathrm{T}$ Tauri stars is not commonly found in the literature. The only publication based on data with spectral resolution and signal-to-noise ratio comparable to those of the data presented in this work is Najita et al. (1996). These authors present $\operatorname{Br} \gamma$ line profiles for five T Tauri stars (DG Tau, AS 353A, V1331 Cyg, AS 205A and S CrA), one Class I source (SVS 13) and one low luminosity embedded object (WL 16). Only two objects (DG Tau and V1331 Cyg) are common to both their and our sample. The Br $\gamma$ line profiles shown by Najita et al. for those two objects are very similar to the ones reported here. The Br $\gamma$ line profiles from the remaining objects presented in Najita et al. have shapes similar to the typical type I or type IV R profiles presented in this work.

Hamann et al. (1988) present high resolution, but relatively low signal-to-noise, $\operatorname{Br} \gamma$ spectra of DG Tau, GW Ori, HL Tau, SU Aur and T Tau, all of which are in the sample studied here. Despite the low signal-to-noise ratio, it can be seen that the line profiles displayed in their Fig. 1 have the same characteristics of the ones presented here. Even SU Aur, seen with a slightly redshifted absorption feature, is somewhat similar to the one shown in our Fig. 2.

Comparison with published line profiles other than the ones mentioned above is very difficult due to the much lower spectral resolutions and signal-to-noise ratios. 
Examples of those are the data shown in Giovanardi et al. (1991) and Evans et al. (1987) where the line profiles are defined by only a few spectral points.

Photometric standard stars or simultaneous photometry is not available for the data set presented here, hence reliable line fluxes could not be determined. Comparison with line fluxes available in the literature is, therefore, not possible. Variability in T Tauri stars, in particular in the NIR magnitudes, implies that any lines fluxes determined using non-simultaneous photometry in conjunction with the measured equivalent widths, would yield unreliable results.

\subsection{Balmer lines}

The results obtained in Sect. 4 for the NIR lines are in complete contrast to what is found for $\mathrm{H} \alpha$ by Edwards et al. (1994), Fernandez et al. (1995), Reipurth et al. (1996) and Alencar \& Basri (2000). Reipurth et al. (1996), who introduce the classification scheme used in this work show that, for a sample of $43 \mathrm{TTS}, 54 \%$ of the $\mathrm{H} \alpha$ line profiles have blueshifted absorptions whereas redshifted absorption features are found in $21 \%$ of the lines. Only $5 \%$ of the profiles are actually classified as type IV R (IPC). The remaining $25 \%$ of the line profiles are classified as type I. As a result the $A f$ of $\mathrm{H} \alpha$ is predominantly less than unity, contrary to what is observed for the NIR lines. The lack of blueshifted absorptions in the NIR lines and the much higher frequency of IPC line profiles are the most prominent differences between $\mathrm{H} \alpha$ and the NIR lines.

Edwards et al. (1994) also present high resolution observations of higher members of the Balmer series (from $\mathrm{H} \beta$ to $\mathrm{H} \delta$ ) for a sample of 15 TTS. These authors show that redshifted absorptions are seen frequently in residual line profiles of the higher Balmer lines (henceforth HBLs). Of the 8 TTS shown in Edwards et al. (1994) to display IPC structure at least in one of the Balmer lines presented there, 5 also display an IPC profile at $\mathrm{Pa} \beta$ and/or at $\mathrm{Br} \gamma$. GM Aur displays IPC structure in $\mathrm{Pa} \beta$ but not in any of the Balmer lines shown in Edwards et al. (1994). Furthermore, for the Balmer lines, both redshifted and blueshifted absorptions can coexist on the same line profile (e.g. DF Tau in Edwards et al. 1994 and Alencar \& Basri 2000). That is not seen in any of the NIR line profiles presented here. The Af of the HBLs falls mostly between 1 and 2.5. Similar values for this parameter are found for $\mathrm{Pa} \beta$ and $\mathrm{Br} \gamma$. The vast majority of the HBLs with redshifted absorption features have them located between about 200 and $300 \mathrm{~km} \mathrm{~s}^{-1}$. Only two of the objects in the Edwards' sample have the centre of the absorption below $200 \mathrm{~km} \mathrm{~s}^{-1}$. When compared to the HBLs, the NIR IPC profiles tend to have the centre of the absorption feature located at lower velocities, often below $200 \mathrm{~km} \mathrm{~s}^{-1}$ (see Tables 6 and 7). Furthermore, the NIR lines analysed here tend to have line wings less extended than those of the HBLs by about $200 \mathrm{~km} \mathrm{~s}^{-1}$. Visual inspection of the Balmer profiles in Edwards et al. (1994) reveal that most line wings extend up to $500 \mathrm{~km} \mathrm{~s}^{-1}$, especially in the blue. From Fig. 6, we see that wings in NIR lines extend typically to about $300 \mathrm{~km} \mathrm{~s}^{-1}$. Also, IPC HBLs tend to have line emission beyond the redshifted absorption feature. In NIR lines, emission seems to stop blueward of the redshifted absorption feature.

In summary, while the NIR lines show very different properties from those of $\mathrm{H} \alpha$, in some aspects they are similar to higher Balmer lines. They are not as wide as the latter, nor seem to be so much influenced by outflowing material. Like the higher Balmer lines, the NIR lines are more prone to IPC structure than $\mathrm{H} \alpha$. The different velocities at which the redshifted absorption feature occurs in IPC higher Balmer lines and in IPC NIR lines should provide constraints on models that hope to explain the formation of hydrogen lines in TTS.

\section{Comparison with accretion/wind models}

A number of hydrogen line profile calculations, within various frameworks, have been done over the years. Hartmann et al. (1990) and Calvet et al. (1992) compute hydrogen line profiles for spherically symmetric wind models and for "cone" geometry inner disk winds respectively. Pedrosa (1996) computed $\mathrm{H} \alpha$ line profiles in the context of a radially spherically symmetric isothermal wind model. Grinin \& Mitskevich (1991) and Mitskevich et al. (1993) drop the assumption of a continuous wind, assume a clumpy structure for the wind and develop the so called "stochastic wind models". Bertout (1977), Bertout (1979) and Bastian (1982) compute line profiles resulting from material radially infalling in spherical or axisymmetric geometry. Calvet \& Hartmann (1992) use a "cone" geometry for the infalling material as a rough approximation to the magnetospheric accretion picture. Hartmann et al. (1994) take a more realistic geometry for infall in the magnetospheric accretion scenario and Muzerolle et al. (1998a) extend the calculations by considering a multilevel atom.

The above models concentrate on computing line profiles for Balmer lines. Explicit model $\mathrm{Pa} \beta$ line profiles are not found in the literature. Results for $\mathrm{Br} \gamma$ lines are found in Hartmann et al. (1990) and in Muzerolle et al. (1998a) only.

The model Br $\gamma$ profile shown in Hartmann et al. (1990) is the result from a non-isothermal wind with a spherically symmetric steady flow (their model 12). Comparing the model line profile with the high resolution observations presented here show no resemblance between them. The model profile peaks at a redshifted velocity larger than $50 \mathrm{~km} \mathrm{~s}^{-1}$ while observed profiles tend to peak at slightly blueshifted velocities. Also, the Af of the model profile is clearly smaller than unity, again in disagreement with the observations. Other near infrared lines computed by Hartmann et al. (1990) (e.g. $\operatorname{Pa} \alpha$ and $\operatorname{Br} \alpha$ ) show either a flat top with the line peak being redshifted or to display a very prominent and broad blueshifted absorption (P Cygni profile), again unlike any of the observed NIR lines shown here.

Muzerolle et al. (1998a) show model $\operatorname{Br} \gamma$ line profiles, arising in a magnetospheric accretion scenario, for 
four different viewing angles: inclinations of 10, 30, 60 and 75 degrees. Full widths at half maximum range from $75 \mathrm{~km} \mathrm{~s}^{-1}$ for the lowest inclination to $110 \mathrm{~km} \mathrm{~s}^{-1}$ for the highest inclination. These are narrower than the typical observed lines, which as we have seen in Sect. 6.2 above, display $F W H M$ mostly between 100 and $300 \mathrm{~km} \mathrm{~s}^{-1}$ with about $60 \%$ of the lines having FWHM between 150 and $250 \mathrm{~km} \mathrm{~s}^{-1}$. Line wings in the model profiles extend to significantly lower velocities than in the observed profiles, especially for the lower inclination models. The 10 and 30 degrees inclination model results show the blue wing extending up to $80 \mathrm{~km} \mathrm{~s}^{-1}$ and the red wing extending to about $125 \mathrm{~km} \mathrm{~s}^{-1}$. In contrast, all of the observed blue wings and most of the observed red wings are significantly more extended than those in the model profiles (see distributions in Fig. 6). Furthermore, contrary to the model predictions, the red wing in observed profiles is not more extended than the blue wing. For the 60 and 75 degrees inclination models (the model IPC profiles) the blue wing extends up to about $170 \mathrm{~km} \mathrm{~s}^{-1}$, while the red wing extends up to $130 \mathrm{~km} \mathrm{~s}^{-1}$. The redshifted absorption feature extends from there up to about $240 \mathrm{~km} \mathrm{~s}^{-1}$. These velocities match reasonably well those observed for the maximum observed velocity of the IPC profiles, but once again, fail at explaining the velocities observed in the blue wing of the profiles. Another difference between the model predicted and the observed profiles lies in the maximum line intensity. Model profiles show normalized intensities of at least about 1.8. Such high intensities are not observed even in TTS with strong $\operatorname{Br} \gamma$ emission. Quantitatively, judging from the model $\mathrm{Br} \gamma$ profiles presented in Muzerolle et al. (1998a), models do not match the observations very well. However, a greater exploration of the model's parameter space is required to assess this matter fully.

From a qualitative standpoint, Muzerolle et al. (1998a)'s results for models with higher inclinations yield $\mathrm{Br} \gamma$ profiles which are IPC in shape and which resemble some of the observed line profiles (e.g. DF Tau, RW Aur), even if in quantitative terms they are different (e.g. in their widths and peak intensities). The double peaked profiles, characteristic of lower inclination models, are not observed at $\mathrm{Br} \gamma$ in the sample of stars discussed here. None of the model Br $\gamma$ profiles presented by Muzerolle et al. (1998a) is a type I profile. These type of profiles constitute the vast majority of the observed ones.

\section{Discussion}

\subsection{NIR lines vs. accretion/wind flows}

The presence of accretion/wind flows in TTS is signaled by redshifted/blueshifted absorption features respectively dipping below the continuum in their line profiles $^{1}$. Whenever $\mathrm{Pa} \beta$ and $\mathrm{Br} \gamma$ display absorption features they are redshifted and dip below the continuum (CW Tau's blueshifted absorption at $\mathrm{Pa} \beta$ is the exception). These features must arise from infalling gas. The

\footnotetext{
${ }^{1}$ Absorptions that never dip below the continuum can also be interpreted as lack of emission.
}

velocities corresponding to those features are in good agreement with those expected for free-falling material from a few stellar radii out. In the context of the magnetospheric accretion scenario, these velocities can be combined with an observational determination of the stellar radius in order to obtain lower limits for the stellar mass. Bonnell et al. (1998) used velocities provided by $\mathrm{Pa} \beta$ and $\operatorname{Br} \gamma$ data presented here to obtain such mass estimates.

Modeling carried out in the context of magnetospheric accretion (e.g. Hartmann et al. 1994) shows that the presence of a redshifted absorption results from seeing the infalling gas against the hot region where the accretion shock occurs. The geometry of the system and the contrast between the temperature of the infalling material and that of the shock region are pivotal in determining whether an absorption appears in the line profile and, if so, the strength of the absorption feature. In fact, they seem to be much more important than the value of the accretion rate itself. It is thus not surprising that plotting accretion rates from Hartigan et al. (1995) and from Gullbring et al. $(1998)^{2}$ against the $E W$ of the absorption feature in the IPC $\mathrm{Pa} \beta$ line profiles shows no significant correlation between the two quantities. Also, no obvious differences in accretion rates are found between stars displaying IPC or type I profiles. As suggested from the modeling, the lack/presence of redshifted absorption in some line profiles does not seem to have an obvious relation with stars accreting matter at different rates or, at least, the accretion rate is not the most important factor determining the actual shape of the line profiles.

To investigate the role of the system's geometry in determining the shape of the line profile we used inclinations available in the literature (Bouvier et al. 1995) for the stars in our sample. From the stars with inclinations in Bouvier et al. (1995), fifteen have $\mathrm{Pa} \beta$ IPC or type I profiles and nine have $\operatorname{Br} \gamma$ IPC or type I profiles. Figure 7 shows the distribution of inclinations for those stars in bins of $30^{\circ}$. These distributions do not show any clear separation in inclination angle for stars with IPC and with type I line profiles. However, the $\mathrm{Pa} \beta$ IPC line profiles tend to occur in systems with $i$ between $30^{\circ}$ and $60^{\circ}$ but rarely above $60^{\circ}$ or below $30^{\circ}$. This is a result obtained from a relatively small number of stars and should be regarded with extreme caution. It should be noted that the magnetospheric accretion model predicts redshifted absorption for systems observed at high inclinations. If systems at intermediate inclinations are really more prone to display IPC structure in their line profiles then the flow geometry in the magnetospheric accretion models envisioned thus far is only a rough approximation of the way in which matter really accretes onto the star. Another indication that

\footnotetext{
2 The accretion rates given by Hartigan et al. (1995) and Gullbring et al. (1998) are both derived from the optical veiling but differ in numerical value due to the different methodology used to transform veiling into accretion luminosity. See Gullbring et al. (1998) for a thorough discussion on the differences between the two approaches.
} 


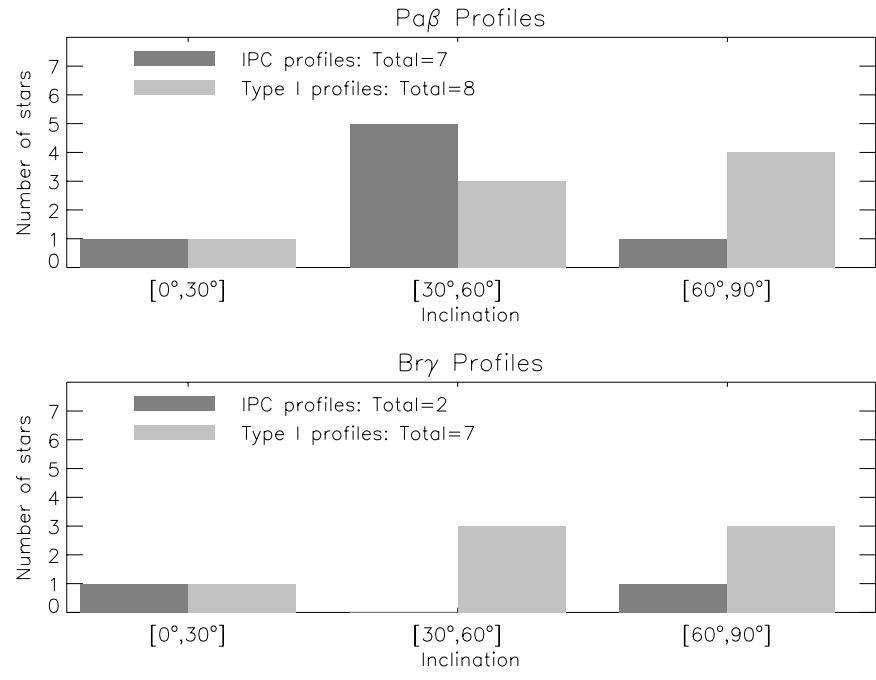

Fig. 7. Distribution of inclinations for IPC and type I profiles. Top panel - $\mathrm{Pa} \beta$; Bottom panel - $\mathrm{Br} \gamma$. Inclinations from Bouvier et al. (1995)
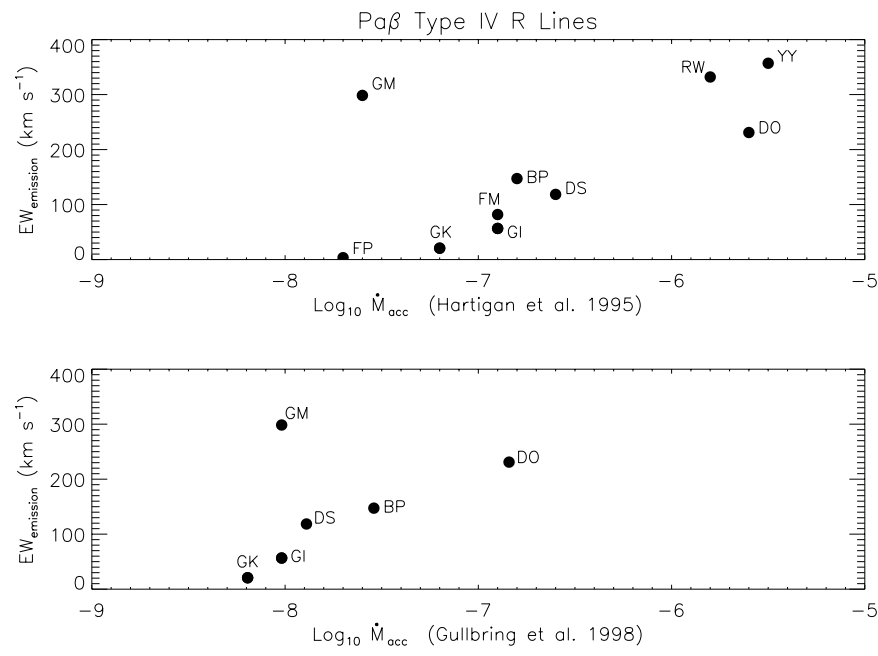

Fig. 8. Accretion Rate vs. emission $E W$ in $\mathrm{Pa} \beta$ IPC lines. Top panel - accretion rates from Hartigan et al. (1995); Bottom panel - accretion rates from Gullbring et al. (1998)

this is the case is the fact that one sees so many type I profiles, which is not predicted by current magnetospheric accretion models.

Since the presence of a redshifted absorption feature is also sensitive to the temperature of the accretion shock, one could, in principle, try to correlate the appearance of IPC structure in a given star with the temperature of its accretion shock. However, the latter is not observationally constrained well enough for individual objects.

What about the origin of line emission? According to Muzerolle et al. (1998a), if hydrogen line emission originates in infalling matter in the context of the magnetospheric accretion model, one should expect to see a correlation between emission line strength and accretion rate.

Plotting accretion/wind rates versus the $E W$ of type I line profiles show no apparent relation between the rate at which mass falls onto or flows away from the stars and

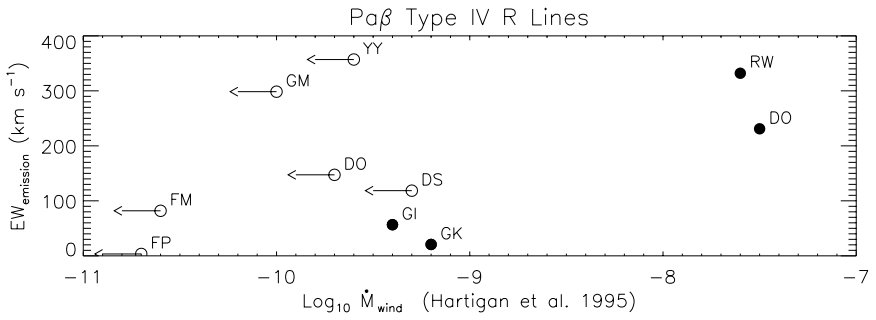

Fig. 9. Wind Mass Loss Rate vs. emission $E W$ in $\mathrm{Pa} \beta$ IPC lines. Mass loss rates from Hartigan et al. (1995)

the strength of the $\mathrm{Pa} \beta$ or $\mathrm{Br} \gamma$ (where the lines strenghts are normalised to the continuum). Pearson's correlation coefficients are 0.44 and 0.41 respectively for $\dot{M}_{\text {acc }}$ vs. $E W$ and $\dot{M}_{\text {wind }}$ vs. $E W$. The number of data points in those plots (not shown) is 16 for $\dot{M}_{\text {acc }}$ vs. $E W$ and 14 for $\dot{M}_{\text {wind }}$ vs. $E W$.

On the other hand, plotting accretion rates versus the $E W$ of the emission component in the $\mathrm{Pa} \beta$ IPC lines (Fig. 8) shows that there seems to be a trend in the sense that stars with larger accretion rates tend to have larger $E W$ in the emission component of the IPC profiles. For this relationship Spearman's $\rho$ rank corrrelation is 0.72 with a significance of 0.008 . A larger number of points in Fig. 8 would be necessary to claim a definite correlation between the quantities in question.

Plotting the wind mass loss rate, as determined by Hartigan et al. (1995), versus the $E W$ of the emission component of the IPC $\mathrm{Pa} \beta$ line profiles hints that no correlation exists between the two quantities (see Fig. 9). This is somewhat surprising given the positive correlation between mass accretion rate and wind mass loss rate observed by Hartigan et al. (1995).

While the amount of emission in IPC profiles does seem to behave according to predictions from existing magnetospheric accretion models, the amount of emission in type I profiles does not seem to follow that trend. One possible explanation for the lack of correlation is that the main determinant of the strength of line emission in these NIR lines is not the amount of accreting matter. Alternatively, radiative transfer and/or geometrical effects may smooth out clear relationships between those two quantities.

Calvet \& Hartmann (1992) were partially motivated by the fact that the Balmer line profiles observed for many $\mathrm{T}$ Tauri stars are generally symmetric and "centrally peaked", i.e. type I profiles, which turned out to be very difficult to explain by wind models. That work and its development (Hartmann et al. 1994) show that infall models in a magnetospheric accretion scenario can produce the desired Balmer line profiles with no obvious signature for accretion. Hartmann et al. (1994) shows that profiles of hydrogen lines arising in magnetospheric accretion flows can be of type I but displaying a distinctive asymmetry due both to geometrical and radiative transfer effects. This asymmetry is in the sense that line profiles have $A$ f's larger than unity and that line peaks occur at slightly blueshifted velocities. 
Edwards et al. (1994) use this asymmetry in Balmer lines as a signature for infall.

As discussed in Sect. 6.2 above, $\mathrm{Pa} \beta$ and $\mathrm{Br} \gamma$ line profiles have their line peaks ocurring at slightly blueshifted velocities and their $A f$ 's are larger than unity (recall Figs. 4 and 5). Applying the same criteria used by Edwards et al. (1994) one can argue that $\mathrm{Pa} \beta$ and $\mathrm{Br} \gamma$ type I profiles result from infall in a magnetospheric accretion scenario. A different geometry and/or different physical conditions in the accretion flow relative to those considered by Hartmann, Calvet, Muzerolle and co-workers seem to be necessary though. The observation of line profile variability implying variable accretion (e.g. Johns \& Basri 1995b) and the presence of localized accretion spots (e.g. Unruh et al. 1998) indeed confirm that axially symmetric accretion models, such as those considered by Hartmann and co-workers, are not truly applicable in $\mathrm{T}$ Tauri stars. The virtue of these models is that they do explain, in a qualitative fashion, the general shape of the observed $\mathrm{Pa} \beta$ and $\mathrm{Br} \gamma$ line profiles. When quantitative comparisons are done, the model $\mathrm{Pa} \beta$ and $\mathrm{Br} \gamma$ lines are too narrow, by about $100 \mathrm{~km} \mathrm{~s}^{-1}$ in both $F W H M$ and $H W Z I$ and also far too intense (by factors of a few in peak intensity).

An alternative to infall models for the origin of line emission are wind models. As thoroughly discussed by Calvet et al. (1992) and by Calvet \& Hartmann (1992), the latter models seem to have great difficulties in explaining type I profiles. With the appropriate choice of parameters, "stochastic" wind models (Grinin \& Mitskevich 1991 and Mitskevich et al. 1993) are able to produce type I profiles for lines of "intermediate optical depth". These were calculated with the intention of comparing the results with observations of the infrared CaII triplet. Detailed calculations, specific for the $\mathrm{Pa} \beta$ and $\mathrm{Br} \gamma$ lines, are needed in order to establish whether a clumpy structure for a wind can produce type I profiles.

As referred to at the beginning of Sect. 3, a number of narrow photospheric absorption lines fall on top of the $\mathrm{Pa} \beta$ emission line (see FE99). These photospheric lines are easily seen in many of the $\mathrm{Pa} \beta$ lines observed. As they are photospheric in origin, the fact they are seen imply that either $\mathrm{Pa} \beta$ is optically thin or, alternatively, that the filling factor of the line emitting region is only a small fraction of the stellar disk, allowing for a direct view of the stellar photosphere.

\section{2. $\mathrm{Pa} \beta$ vs. $\mathrm{Br} \gamma$}

How different is the information conveyed by $\mathrm{Pa} \beta$ with respect to that given by $\mathrm{Br} \gamma$ ?

Almost half of the stars in the studied sample with line emission either at $\mathrm{Pa} \beta$ or $\mathrm{Br} \gamma$ have both lines displaying similar characteristics. The most conspicuous differences are stars that have IPC profiles at $\mathrm{Pa} \beta$ but type I at $\mathrm{Br} \gamma$ or that display emission at $\mathrm{Pa} \beta$ but no emission at all at $\mathrm{Br} \gamma$. The former can result either from the physical conditions where the lines are formed or from the non-simultaneity of the $\mathrm{Pa} \beta$ and $\mathrm{Br} \gamma$ line profiles (they were obtained one day apart). $\operatorname{Br} \gamma$ lines that have type I profiles when the corresponding $\mathrm{Pa} \beta$ are IPC tend to have $A f$ 's larger than the average for their class, with more emission blueward of the line rest velocity. This tends to point to the former explanation, with $\mathrm{Br} \gamma$ optically thinner than $\mathrm{Pa} \beta$ and hence less prone to display redshifted absorptions. If this is the case, it can provide constraints on the physical conditions of the infalling gas.

Lack of detected $\mathrm{Br} \gamma$ emission while $\mathrm{Pa} \beta$ is in emission shows that for some stars the physical conditions in the hydrogen gas that surrounds them are such that population of level 7 is not too significant while a reasonable amount of atoms have level 5 populated.

A significant percentage of the $\mathrm{Br} \gamma$ IPC lines tend to peak more towards the blue than the $\mathrm{Pa} \beta$ IPC lines (Sect. 6.2 above). The blueward shift is of about $60 \mathrm{~km} \mathrm{~s}^{-1}$. It is worthwhile noting that amongst the $\mathrm{Br} \gamma$ type I line profiles a significant number also have the velocity of the line peak shifted to the blue relative to $v_{\text {peak }}$ in the $\mathrm{Pa} \beta$ lines (see Fig. 4). An explanation for this shift would be that, due to their different optical depths, $\mathrm{Pa} \beta$ and $\mathrm{Br} \gamma$ sample regions where gas moves at different speeds, resulting in line profiles peaking at different velocities. Surprisingly the magnetospheric accretion line profile modeling carried out by Hartmann, Calvet, Muzerolle et al. does not show any relative shift in the peak velocity between lines of very different optical depths, such as $\mathrm{H} \alpha$, $\mathrm{H} \beta, \mathrm{H} \gamma$, and $\mathrm{Br} \gamma$.

\subsection{Multiple systems}

Multiplicity amongst the population of the T Tauri stars is well known (Ghez et al. 1993; Leinert et al. 1993; Richichi et al. 1994; Mathieu 1994; Simon et al. 1995). The separation between components in many of those systems is such that the NIR lines observed might be influenced by the presence of the companion stars. From the 50 stars in the sample presented in this work, the following 16 are, according to the references above, binary or multiple systems with separation between components smaller than the slit width used for the observations reported here. They are: DD Tau, DF Tau, DQ Tau, FS Tau, FX Tau, GG Tau, GH Tau, HP Tau, LkCa7, RW Aur, T Tau, UY Aur, V773 Tau, VY Tau, XZ Tau and ZZ Tau. Of these 16 binary/multiple stars, 9 have $\mathrm{Pa} \beta$ in emission, from which 6 are of type I, and 3 are IPC profiles; from the 6 displaying $\mathrm{Br} \gamma$ in emission, 5 are of type $\mathrm{I}$ and 1 is an IPC profile. Amongst the T Tauri stars that are part of a multiple system type I line profiles are the most common, followed by the IPC profiles. This is in tune with the results obtained for the whole sample. Excluding the binary/multiple systems from the classification scheme does not change the results significantly.

\subsection{Line profile variability}

A few TTS for which spectra are presented in this work were observed more than once and some considerations 
regarding variations in the observed NIR line profiles are due. The stars for which the NIR lines were observed more than once are shown in Table 8 where the dates of the observations are also indicated.

Observations in consecutive nights of the same line for the same star were only carried out during the December 1995 run, only at $\mathrm{Pa} \beta$ line and only for two nights. The night to night variation observed is not very dramatic, in the sense that, for these stars, the type of line profile did not change, despite changes in equivalent width and/or in the position of absorption features.

For some of the stars, more drastic changes are observed to occur in spectra taken roughly one year apart. DR Tau changes from type I into type II R and GK Tau changes from type I into an IPC line profile. The $\mathrm{Br} \gamma$ lines of DR Tau and GG Tau and the $\mathrm{Pa} \beta$ line of GI Tau change significantly in terms of equivalent width (due to line profile variations) but preserve the type of profile.

The type of variations that can occur in NIR lines are diverse and must reflect the dynamical activity and/or changes in the physical characteristics of the emitting region(s) (the change of the $\mathrm{Pa} \beta$ profile of GK Tau from type I to IPC surely indicates either that an accretion region came into view or that accretion activity in the star changed between the first and second observing runs). For a given star, monitoring these lines over at least two rotation periods (typically a week to a week and a half) would tell us how the infalling matter behaves, since any variability displayed, especially in a redshifted absorption component, should be associated with an accretion flow. As an example, if a magnetospheric accretion scenario is correct and the rotation axis of the star is tilted relative to the magnetic axis one expects to find a correlation between the line profiles and the phase of the rotation period of the star (Johns \& Basri 1995b). A better understanding of how and where these lines are formed in TTS certainly requires a variability study of these lines.

\section{Conclusion}

From the $\mathrm{Pa} \beta$ and $\mathrm{Br} \gamma$ observations of a sample of 50 $\mathrm{T}$ Tauri stars we conclude that blueshifted absorptions in the profiles of those lines are very rare. Of the 42 stars observed to have $\mathrm{Pa} \beta$ in emission only one shows a blueshifted absorption, while of the 30 displaying $\operatorname{Br} \gamma$ in emission none has a blueshifted absorption. The most common profiles are of type I $(54 \%$ at $\mathrm{Pa} \beta$ and $73 \%$ at $\mathrm{Br} \gamma$ ). IPC line profiles constitute $34 \%$ of the $\mathrm{Pa} \beta$ profiles and $20 \%$ of the $\operatorname{Br} \gamma$ profiles. These figures are in complete contrast to those of $\mathrm{H} \alpha$, the most studied hydrogen emission line in TTS. Comparison with higher members of the Balmer series reveal some similarities between the two sets of lines, especially in that they are more prone to display IPC structure than $\mathrm{H} \alpha$.

The $\mathrm{Pa} \beta$ and $\operatorname{Br} \gamma$ lines are very wide $(F W H M \approx$ $200 \mathrm{~km} \mathrm{~s}^{-1}$ ), slightly blueshifted and with line wings extending to about $300 \mathrm{~km} \mathrm{~s}^{-1}$ in the blue and to about $200 \mathrm{~km} \mathrm{~s}^{-1}$ in the red. The Asymmetry Factor $(A f)$ is slightly larger than one for most cases, with the $\mathrm{Br} \gamma$
Table 8. Stars for which the NIR lines were observed more than once. The day when an observation was made, is marked by either " $\mathrm{Pa} \beta$ " or " $\mathrm{Br} \gamma$ "

\begin{tabular}{c|cccc|ccc}
\hline \hline Star & \multicolumn{5}{|c|}{$\mathrm{UT9410}$} & \multicolumn{3}{c}{$\mathrm{UT9512}$} \\
& 02 & 03 & 04 & 05 & 15 & 16 & 17 \\
\hline DG Tau & - & $\mathrm{Pa} \beta$ & - & - & $\mathrm{Pa} \beta$ & $\mathrm{Pa} \beta$ & - \\
DL Tau & - & $\mathrm{Pa} \beta$ & - & - & $\mathrm{Pa} \beta$ & $\mathrm{Pa} \beta$ & - \\
DR Tau & - & $\mathrm{Pa} \beta$ & - & - & $\mathrm{Pa} \beta$ & $\mathrm{Pa} \beta$ & - \\
& - & $\mathrm{Br} \gamma$ & - & - & - & - & $\mathrm{Br} \gamma$ \\
GG Tau & - & - & - & $\mathrm{Br} \gamma$ & - & - & $\mathrm{Br} \gamma$ \\
GI Tau & $\mathrm{Pa} \beta$ & - & - & - & $\mathrm{Pa} \beta$ & - & - \\
& - & $\mathrm{Br} \gamma$ & - & - & - & - & $\mathrm{Br} \gamma$ \\
GK Tau & $\mathrm{Pa} \beta$ & - & - & - & $\mathrm{Pa} \beta$ & - & - \\
& - & $\mathrm{Br} \gamma$ & - & - & - & - & $\mathrm{Br} \gamma$ \\
RW Aur & - & - & - & - & $\mathrm{Pa} \beta$ & $\mathrm{Pa} \beta$ & - \\
& - & - & - & $\mathrm{Br} \gamma$ & - & - & $\mathrm{Br} \gamma$ \\
RY Tau & - & - & - & - & $\mathrm{Pa} \beta$ & $\mathrm{Pa} \beta$ & - \\
SU Aur & - & - & - & - & $\mathrm{Pa} \beta$ & $\mathrm{Pa} \beta$ & - \\
\hline \hline
\end{tabular}

distribution for this parameter showing a larger spread than that of the $\mathrm{Pa} \beta$ distribution. The former also displays larger $A f \mathrm{~s}$ than the latter. A significant number of IPC $\mathrm{Br} \gamma$ lines are displaced to the blue by about $50 \mathrm{~km} \mathrm{~s}^{-1}$ relative to the IPC $\mathrm{Pa} \beta$ lines.

If the line emitting region for $\mathrm{Pa} \beta$ sits in front of the whole stellar disk, the line is optically thin. Alternatively, the filling factor of the line emitting region is small and the stellar photosphere is also directly observed.

Comparing the data presented here and the results available in the literature from models for the formation of the hydrogen lines reveal that both wind and accretion models fail to explain the observed line profiles in most cases. The accretion models, computed in the context of the magnetospheric accretion scenario, do provide a qualitative insight on how these lines might be produced in the T Tauri stars' environment but fail under a quantitative comparison. The models produce lines too narrow (by $\sim 100 \mathrm{~km} \mathrm{~s}^{-1} F W H M$ ), with wings extending to velocities too small (by at least $\sim 100 \mathrm{~km} \mathrm{~s}^{-1}$ ) and with far too high maximum normalized intensities (by factors of a few). If nothing else, the discrepancies found between observations and model line profiles hint that the axi-symmetric models considered thus far are just a rough approximation to the real accretion flows in $\mathrm{T}$ Tauri stars.

The redshifted absorption feature in the $\mathrm{Pa} \beta$ and $\mathrm{Br} \gamma$ IPC profiles must be formed in infalling material. It is located at velocities of the order of the free-fall velocity from a few radii out for a typical $\mathrm{T}$ Tauri star. There seems to be a trend associating the amount of emission seen in the IPC profiles and accretion rates, suggesting that lines with this type of profile originate mostly, if not completely, from infalling material. A similar trend does not seem to be present for type I profiles. However, with the exception of the lack of redshifted absorption feature, the latter display similar characteristics to the IPC profiles. In particular, they are centrally peaked and slightly blueshifted, characteristics that are very difficult to obtain in wind models (Calvet \& Hartmann 1992) but arise 
naturally in inflow models due to absorption of infalling redshifted material.

Continuous wind models tend to produce lines with normal $\mathrm{P}$ Cygni profiles but $\mathrm{Pa} \beta$ or $\mathrm{Br} \gamma$ calculations are seldom found in the literature. Stochastic wind models might be able to produce profiles similar to type I but there are no specific predictions for the $\mathrm{Pa} \beta$ and $\mathrm{Br} \gamma$ lines. The way in which winds affect the shape of these NIR lines should be investigated further.

The data set presented here demonstrate that current knowledge about the formation of hydrogen lines in $\mathrm{T}$ Tauri stars is far from providing a detailed explanation for their characteristics and origin. Also, it provides a solid database with which model results can be compared. Hydrogen lines constitute one of the most important diagnostics available for the study of $\mathrm{T}$ Tauri stars and understanding their origin is of crucial importance. From a theoretical point of view, models have to simultaneously explain the near infrared lines and the Balmer lines, which as we have seen above convey different information. An observational effort to try to understand how and why the lines vary is also very important. Otherwise, we will only be trying to understand $\mathrm{T}$ Tauri stars and what gives rise to hydrogen emission lines from a single snapshot of what is, in reality, an ever changing system.

Acknowledgements. We thank the referee, Dr. Suzan Edwards, for insightful comments that significantly improved this paper. D.F.M. Folha acknowledges financial support from the "Subprograma Ciência e Tecnologia do $2^{\circ}$ Quadro Comunitário de Apoio". This research has made use of the Simbad database, operated at CDS, Strasbourg, France. The United Kingdom Infrared Telescope, is operated by Joint Astronomy Centre on behalf of the U.K. Particle Physics and Astronomy Research Council.

\section{References}

Alencar, S. H. P., \& Basri, G. 2000, AJ, 119, 1881

Bastian, U. 1982, A\&A, 109, 245

Bertout, C. 1977, A\&A, 58, 153

Bertout, C. 1979, A\&A, 80, 138

Bonnell, I. A., Smith, K. W., Meyer, M. R., et al. 1998, MNRAS, 229, 1013

Bouvier, J., Covino, E., Kovo, O., et al. 1995, A\&A, 299, 89

Calvet, N., Hartmann, L., \& Hewett, R. 1992, ApJ, 386, 229

Calvet, N., \& Hartmann, L. 1992, ApJ, 386, 239

Camenzind, M. 1990, Rev. Mod. Astron., 3, 234

De Campli, W. M. 1981, ApJ, 244, 124

Edwards, S., Cabrit, S., Strom, S. E., et al. 1987, ApJ, 321, 473

Edwards, S., Hartigan, P., Ghandour, L., \& Andrulis, C. 1994 , AJ, 108, 1056

Evans II, N. J. E., Levreault, R. M., Beckwith, S., \& Skrutskie, M. 1987, ApJ, 320, 364

Feigelson, E. D., Welty, A. D., Imhoff, C., et al. 1994, ApJ, 432,373

Fernandez, M., Ortiz, E., Eiroa, C., \& Miranda, L. F. 1995, A\&AS, 114, 439
Folha, D. F. M. 1998, Ph.D. Thesis, Queen Mary \& Westfield College, University of London, United Kingdom (Available On-line at URL: www . astro.up.pt/users/dfmf/Thesis/thesis.html)

Folha, D. F. M., \& Emerson, J. P. 1999, A\&A, 352, 517

Gameiro, J. F., Lago, M. T. V. T., Lima, N. M., \& Cameron, A. C. 1993, MNRAS, 261, 11

Ghez, A. M., Neugebauer, G., Matthews, K. 1993, AJ, 106, 2005

Giovanardi, C., Gennari, S., Natta, A., \& Stanga, R. 1991, ApJ, 367, 173

Grinin, V. P., \& Mitskevich, A. S. 1991, Ap\&SS, 185, 105

Guenther, E., \& Hessman, F. 1993a, A\&A, 268, 192

Guenther, E., \& Hessman, F. 1993b, A\&A, 276, L25

Gullbring, E., Hartmann, L., Briceño, C., \& Calvet, N. 1998, ApJ, 492, 323

Hamann, F., Simon, M., \& Ridgway, S. T. 1988, ApJ, 326, 859

Hartigan, P., Edwards, S., \& Ghandour, L. 1995, ApJ, 452, 736

Hartmann, L., Edwards, S., \& Avrett, E. H. 1982, ApJ, 261,279

Hartmann, L., Calvet, N., Avrett, E. H., \& Loeser, R. 1990, ApJ, 349, 168 (HCAL90)

Hartmann, L., \& Kenyon, S. J. 1990, ApJ, 349, 190

Hartmann, L., Hewett, R., \& Calvet, N. 1994, ApJ, 426, 669

Herbig, G. H., \& Bell, K. R. 1988, Third Catalogue of EmissionLine Stars of the Orion Population, Lick Observatory Bulletin Series No. 111, Lick Observatory, Board of Studies in Astronomy \& Astrophysics, Univ. of California, Santa Cruz

Horne, K. 1986, PASP, 98, 609

Johns, C. M., \& Basri, G. 1995a, AJ, 109, 2800

Johns, C. M., \& Basri, G. 1995b, ApJ, 449, 341

Kitamura, Y., Kawabe, R., \& Saito, M. 1996, ApJ, 457, 277

Königl, A. 1991, ApJ, 370, L39

Lago, M. T. V. T. 1979, Ph.D. Thesis, University of Sussex, Falmer, UK

Leinert, C., Zinnecker, H., Weitzel, N., et al. 1993, A\&A, 278, 129

Levreault, R. M. 1988, ApJS, 67, 283

Mathieu, R. D. 1994, ARA\&A, 32, 465

Mitskevich, A. S., Natta, A., \& Grinin, V. P. 1993, ApJ, 404, 751 (MNG93)

Muzerolle, J., Calvet, N., \& Hartmann, L. 1998, ApJ, 492, 743 (MCH98)

Muzerolle, J., Hartmann, L., \& Calvet, N. 1998, AJ, 116, 455

Najita, J., Carr, J. S., \& Tokunaga, A. T. 1996, ApJ, 456, 292

Natta, A., Giovanardi, C., \& Palla, F. 1988, ApJ, 332, 921

Neuhaeuser, R., Sterzik, M. F., Schmitt, J. H. M. M., Wichmann, R., \& Krautter, J. 1995, A\&A, 297, 391

Pedrosa, A. 1996, Ph.D. Thesis, Faculty of Sciences, Porto University, Porto, Portugal

Puxley, P. J., Beard, S. M., Ramsey, S. K. 1992, ed. P. Grosbøl, \& R. de Ruijsscher, 4th ESO/ST-ECF Data Analysis Workshop, ESO Conf. Works. Proc. 41, 117

Reipurth, B., Pedrosa, A., \& Lago, M. T. V. T. 1996, A\&A, 20, 229

Richichi, A., Leinert, C., Jameson, R., Zinnecker, H. 1994, A\&A, 287, 145

Simon, M., Ghez, A. M., Leinert, C., et al. 1995, ApJ, 443, 625

Shu, F. H., Najita, J., Ostriker, E., et al. 1994, ApJ, 429, 781

Unruh, Y. C., Cameron, A. C., \& Guenther, E. 1998, MNRAS, 295,781 